\title{
Phenomenological Force and Swelling Models for Rechargeable Lithium-ion Battery Cells
}

\author{
Ki-Yong Oh, Bogdan I. Epureanu*, Jason B. Siegel, and Anna G. Stefanopoulou \\ Department of Mechanical Engineering, University of Michigan \\ 2350 Hayward Street, Ann Arbor, MI 48109-2125, USA
}

*Corresponding author: Tel.: +1-734-647-6391; fax: +1-734-615-6647

E-mail address: epureanu@umich.edu

\begin{abstract}
Three phenomenological force and swelling models are developed to predict mechanical phenomena caused by Li-ion intercalation: a 1-D force model, a $1^{\text {st }}$ order relaxation model, and a 3-D swelling model. The 1-D force model can estimate the Li-ion intercalation induced force for actual pack conditions with preloads. The model incorporates a nonlinear elastic stiffness to capture the mechanical consequences of Li-ion intercalation swelling. The model also separates the entire state of charge range into three regions considering phase transitions. The $1^{\text {st }}$ order relaxation model predicts dynamic swelling during relaxation periods. A coefficient of relaxation is estimated from dynamic and quasi-static swelling at operational conditions. The 3-D swelling model predicts the swelling shape on the battery surface for all states of charge. This model introduces an equivalent modulus of elasticity, which is dependent on the state of charge, to
\end{abstract}


capture material transformations of the electrodes, and the orthotropic expansion of the jellyroll in a direction perpendicular to the electrode surfaces. Considering the simplicity of the measurements and direct physical correlations between stress and strain, the proposed models can enhance battery management systems and power management strategies.

\section{Keywords}

Li-ion battery; Li-ion intercalation; swelling; phenomenological model; volume change

\section{Introduction}

Volatility of oil prices, diminishing natural resources, and climate change are triggering many countries to investigate ways to reduce energy consumption. These trends motivate automobile industries to concentrate on the development of eco-friendly, high-efficiency vehicles. With the growing market for electrified vehicles, attention for Lithium-ion (Li-ion) batteries has increased because Li-ion batteries are reversible power sources used in electrified vehicles. This trend can be attributed mainly to their ability to combine a high gravimetric/volumetric energy and power density, which leads to compact and low-weight batteries [1-3]. Moreover, low self-discharge rate and long cycle-life make Li-ion batteries widely popular in portable electronics. Indeed, Liion batteries are highly versatile energy storage devices for a variety of applications from smallscale portable electronics to large-scale electrified vehicles.

Recent market demands for advanced Li-ion batteries emphasize not only high-energy/power density but also improved reliability and safety for the application of electric vehicles (EVs) and 
hybrid electric vehicles (HEVs) due to the stringent safety standards for air and ground applications [4]. The safety and reliability of Li-ion batteries, both of which are critical for the development of EVs and HEVs, can be improved with accurate battery models combined with novel battery management strategies. Thus, there have been considerable efforts to develop battery models to depict the dynamics of Li-ion batteries. These include electrical equivalent circuit models [5,6], electro-chemical models [7], and the heat transfer models [8-11]. Moreover, the exploitation of various control methods and strategies for battery management system (BMS) is increasing [12-14].

The effects of stress caused by Li-ion intercalation/deintercalation and the effects of mechanical loads on the cell performance depend on physics ranging from micro-scale to macro-scale. The mechanical fatigue associated with cycling may lead to performance degradation, capacity loss, and eventual failure [15-20]. The effects of prestress and stress evolution on the fading of the battery capacity over time/cycling have been studied through experiments [21,22]. This suggests that mechanical damage in electrodes is driven by the stress and strain fields that are induced from repeated cycling. These studies have provided a useful foundation for developing fracture criteria and characterizing the relationship between mechanical forces and Li-ion intercalation during charge and discharge cycles. In contrast to micro-scale [23-25], the macro-scale stress and strain responses are directly observable and measurable with high accuracy. This suggests that the development of phenomenological battery models can improve the safety and reliability of batteries, and eventually enhance the lifespan and capability of battery cells and packs.

This paper proposes three phenomenological force and swelling models for Li-ion batteries. The 1-D force model includes a nonlinear elastic stiffness to capture the inherent mechanical consequences of Li-ion intercalation; the measured force not only varies with the state of charge 
significantly but also shows significant nonlinear characteristics with respect to the amplitude of Li-ion intercalation swelling. Moreover, this model recognizes three separate SOC regions delimited by phase transitions. The $1^{\text {st }}$ order relaxation model predicts dynamic (transient) swelling during relaxation periods by using a visco-elastic mechanical relaxation approach. The 3-D swelling model uses a high fidelity finite element model of the battery. This 3-D swelling model accounts for the dependence of an equivalent modulus of elasticity on the state of charge (SOC). Also, the model captures the orthotropic expansion in a direction perpendicular to the electrode surfaces. Therefore, this model can predict the swelling shape on the battery surface over SOC. Validation was accomplished by comparing predicted overall free swelling shapes with measurements at a variety of SOCs. This model is useful to predict the overall swelling shape and the magnitude of swelling at a certain location during actual operational conditions, i.e. constrained conditions. The three models proposed herein can be used to improve existing battery management systems by enabling novel power management schemes as suggested in [51].

\section{Experimental Set-up and Measurements}

This study used a flat-wound type prismatic 5Ah Li-ion battery cell. Detailed information of the Li-ion battery cell and experimental set-up is available in [26,27].

In a first experiment, dynamic (transient) free swelling was measured over SOC. The net displacement at the center of the battery (location 13 in Figure 1) was measured with respect to SOC during discharge using a standard constant current discharging profile. Prior to discharge, 
the battery was fully charged using a CCCV protocol at $2 \mathrm{~A}(0.4 \mathrm{C})$ and rested 3 hours at a fixed temperature of $25^{\circ} \mathrm{C}$ regulated by a thermal chamber. The voltage was clamped after reaching $4.1 \mathrm{~V}$, at which time it was held until the current tapered to $\mathrm{C} / 100(50 \mathrm{~mA})$. Discharge was performed down to $3.0 \mathrm{~V}$ at $1.0 \mathrm{~A}(0.2 \mathrm{C})$. Measurements with a thermocouple showed that the applied current did not cause significant heating without thermal swelling. Also, the battery cell surface remained within $0.1{ }^{\circ} \mathrm{C}$ from the regulated $25^{\circ} \mathrm{C}$ ambient temperature during discharge. The swelling at $0.2 \mathrm{C}$ therefore allows direct correlations to be made between swelling and Li-ion intercalation in a cell sandwich without significant convolution by thermal expansion. Note that discharge at a low C-rate is essential to obtain pure Li-ion intercalation swelling because discharge at a high C-rate is accompanied by thermal swelling [26].

In a second experiment, the quasi-static free swelling was measured over SOC. In this experiment, the relative expansion in the $z$ direction (perpendicular to the electrodes), was measured at 5 locations labeled as 7, 9, 13, 17, and 19 in Figure 1. Prior to discharge, the battery was fully charged using a CCCV protocol. To obtain the desired SOC ranging from $0 \%$ to $100 \%$ with $5 \%$ increments, the battery was incrementally discharged at a $0.4 \mathrm{C}$ rate $(0.4 \mathrm{C}$ current of actual capacity) with an appropriate time (7.5 minutes). Each incremental discharge was followed by 3 hours of rest time to ensure that the system reached equilibrium after each incremental discharge.

In a third experiment, swelling at the fully charged state was measured with sensors sequentially placed at locations 1 to 25 in Figure 1. This experiment used the same protocol as the first experiment except for the C-rate which was $0.4 \mathrm{C}$. The swelling at the fully charged state was measured after 3 hours of rest time to ensure that thermal swelling due to the increased C-rate did not affect the measurements. 
In a fourth experiment, the quasi-static force was measured over SOC with another experimental set-up. Details of the experimental set-up can be found in [28]. Specifically, forces created in a pack of 3 cells were measured during quasi-static charge and discharge with 3 different initial preloads. The battery pack of interest consists of dozens of batteries connected in series, and plastic spacers are placed between cells [28]. To mimic these conditions, the experimental set-up consists of 3 nominally identical cells connected in series and mechanically sandwiched between two 1 -inch thick garolite plates assembled with 4 bolts at their corners. Each bolt was instrumented with a load sensor (LC8150-250-100, USA). The entire set-up was placed inside a thermal chamber (Cincinnati Sub-Zero ZPHS16-3.5-SCT/AC, USA) that controls the desired temperature $\left(25^{\circ} \mathrm{C}\right)$. The plastic spacers between cells allow air to flow between batteries while also constraining the batteries from expanding. Hence, the force measured in this set-up can represent the force induced from the volume change of batteries in an actual battery pack.

\section{Phenomenological 1-D force model}

\subsection{Model description}

Two different experiments were carried out to characterize the quasi-static response of the battery cell in section 2, namely the swelling versus SOC and the force versus SOC at steady state thermal equilibrium. The relationship between force and swelling can be determined when such two experiments are used together (and the curve of force versus swelling is parameterized by the SOC). However, experimental conditions were different; the swelling was measured at 
free conditions without the plastic spacer and with no preload, while the reaction force was measured at constrained conditions with the plastic spacer and with preload. Hence, governing equations have to be obtained to couple these two experiments considering these differences. These equations are derived in this section. Based on governing equations, equivalent stiffness values are obtained for the case, jellyroll, and spacer. Next, the force is predicted at actual operational/pack conditions.

Three different conditions are considered to describe the experiments. These conditions represent the quasi-static behavior of: (a) the battery cell at free conditions during Li-ion intercalation; (b) the swelling of battery cell outfitted with a spacer where a constant preload was applied; and (c) the force applied from tightening the bolts of the fixture constrains the battery cell and spacer with a constant preload during Li-ion intercalation. It is assumed that the equivalent stiffness at free and constrained conditions is approximately the same.

Figure 2 (a) depicts the quasi-static behavior of the battery cell at free conditions, i.e. the measurement of free swelling without any constraint during Li-ion intercalation. This experiment is conducted with a battery cell which consists of the case and the jellyroll. Thus, two equivalent stiffness elements are modeled: the equivalent stiffness $k_{a_{\bullet}}$ of the active material (jellyroll), and the equivalent stiffness $k_{c}$ of the case. Both these equivalent stiffness values refer to the relationship between the total force applied on the cell and the deflection of the cell in a direction perpendicular to the electrodes. Note that these two stiffness elements are connected in parallel (and not in series) because the case restricts the expansion of the jellyroll. A nonlinear model is used for the jellyroll [36] to account for the dependence of force on swelling as 


$$
F_{a}=k_{a_{1}} s_{a}^{f}+k_{a_{2}}\left(s_{a}^{f}\right)^{3},
$$

where $F_{a}$ is the force in the jellyroll created by Li-ion intercalation, while $k_{a_{1}}$ and $k_{a_{2}}$ are the linear and nonlinear equivalent stiffness values. $s_{a}^{f}$ is the swelling of the jellyroll when the jellyroll is not constrained by the case, i.e. the expansion of the jellyroll without any constraints. Superscript $f$ denotes free swelling conditions.

Consider as initial state the fully delithiated state when no force is exerted in the battery cell. When the battery is charged, Li-ions move from cathode to anode. This process leads to a volume expansion of the jellyroll (which leads to the swelling $s_{a}^{f}$ ). During $\mathrm{Li}$-ion intercalation, the case impedes the expansion of the jellyroll when the jellyroll is placed inside of the case due to the stiffness of the case. Thus, the equivalent swelling of the battery cell (denoted as $s_{c}^{f}$ in Figure 2 (a)) might be smaller than the swelling of the jellyroll without the case.

At the equilibrium state, the jellyroll is compressed, while the case is stretched from their original size because the volume of the jellyroll changes due to Li-ion intercalation. Hence, different forces are induced into each component. A compressive force acts on the jellyroll, whereas a tensile force acts on the case. The resultant force is zero at mechanical equilibrium. Thus, the compression force which acts on the jellyroll $\left(F_{a}\right.$ in Figure $\left.2(a)\right)$ is the same as the compressive force which acts on the case $\left(F_{c}\right.$ in Figure 2 (a)). The superscript $c$ denotes constrained swelling conditions. The deformation of the case is $s_{c}^{f}$ and the deformation of the jellyroll is $s_{a}^{f}-s_{c}^{f}$ in a direction perpendicular to electrodes at the balanced position. Using Eq. (1), the balance of forces gives 


$$
k_{c} s_{c}^{f}=k_{a_{1}}\left(s_{a}^{f}-s_{c}^{f}\right)+k_{a_{2}}\left(s_{a}^{f}-s_{c}^{f}\right)^{3} .
$$

Figure 2 (b) describes the initial assembly of the force experimental setup where the Li-ion battery cell is assembled with a spacer. Therefore, one more stiffness element which depicts the equivalent stiffness of the spacer $\left(k_{s}\right)$ in a direction perpendicular to electrodes is added in series. An external load $\left(F_{0}\right)$ is applied to the battery in the experiment by tightening the bolts of the fixture to replicate conditions experienced in a battery pack. This external load results in initial displacements for the spacer and the case $\left(s_{0}\right.$ and $s_{0_{c}}$ in Figure $\left.2(\mathrm{~b})\right)$. Thus, the total deformation of the spacer is $s_{0}-s_{0_{c}}$. At equilibrium, a compression force acts upon all elements due to the external load. The compression force which acts on the spacer is the external load. Thus, $F_{0}=F_{s}$ as shown in Figure 2 (b). This load is also the same as the sum of forces which act on the case and jellyroll. Thus, $F_{0}=F_{c}+F_{a}$ as shown in Figure 2 (b). Equating the compression force acting on the spacer with the compression force acting on the case and the jellyroll, one obtains

$$
k_{s}\left(s_{0}-s_{0_{c}}\right)=k_{c} s_{0_{c}}+k_{a_{1}} s_{0_{c}}+k_{a_{2}} s_{0_{c}}^{3}
$$

Figure 2 (c) illustrates the force measurement during Li-ion intercalation at constrained conditions. In this figure, the battery cell and spacer are fixed on both sides by two rigid end plates with a preload. Then, the charge state of the battery cell is changed and the reaction force is measured by the load cells. The unconstrained swelling of the jellyroll $s_{a}^{f}$ shown in Figures 2 (a) and 2 (c) is the same because it is the swelling of the jellyroll without any constraints. However, the swelling of the jellyroll and case $\left(s_{c}^{c}\right)$ is different than the swelling of the jellyroll 
at free conditions $\left(s_{c}^{f}\right)$ because of the stiffness of the spacer and because of the fixed conditions. During operation in constrained conditions, the case is stretched (similar to the free swelling conditions) while the jellyroll and spacer are compressed. The stretching of the case is $s_{c}^{c}$, whereas the compression of the jellyroll and spacer are $s_{a}^{f}-s_{c}^{c}$ and $s_{c}^{c}+s_{0}$ respectively. Note that the initial displacement of the spacer due to the preload should be considered because the charge/discharge process is conducted with preload. Therefore, a tensile force acts on the case, whereas a compressive force acts on the jellyroll and spacer. The force acting on the jellyroll equals the sum of the forces acting on the case and spacer, as shown in Figure $2(\mathrm{c}): F_{s}+F_{c}=F_{a}$. This force equilibrium results in

$$
k_{s}\left(s_{c}^{c}+s_{0}\right)+k_{c} s_{c}^{c}=k_{a_{1}}\left(s_{a}^{f}-s_{c}^{c}\right)+k_{a_{2}}\left(s_{a}^{f}-s_{c}^{c}\right)^{3} .
$$

The measurement location for the force is the side wall (shown as a filled square in Figure 2 (c)). The load cells are placed between the rigid end plate and the heads of the bolts. Hence, the measured force is the same to the compression force acting on the spacer. One obtains

$$
\begin{aligned}
F_{m} & =k_{a_{1}}\left(s_{a}^{f}-s_{c}^{c}\right)+k_{a_{2}}\left(s_{a}^{f}-s_{c}^{c}\right)^{3}-k_{c} s_{c}^{c} \\
& =k_{s}\left(s_{c}^{c}+s_{0}\right)
\end{aligned}
$$

Equation (2) defines the relationship between the unconstrained swelling of the jellyroll $\left(s_{a}^{f}\right)$ and the swelling measured at the center of the case $\left(s_{c}^{f}\right)$ without any constraints. Using this equation, the swelling of the jellyroll can be obtained in terms of the measured swelling of the case at free conditions as $s_{a}^{f}=f\left(s_{c}^{f} ; k_{a_{1}}, k_{a_{2}}, k_{c}\right)$ (where $f$ is a known function). 
Equation (3) defines the relationship between the initial displacement of the spacer $\left(s_{0}\right)$ and that of the battery cell $\left(s_{0_{c}}\right)$ due to the external load. Using this equation, the initial displacement of the spacer can be expressed in terms of that of the case (battery cell) as $s_{0}=g\left(s_{0_{c}} ; k_{a_{1}}, k_{a_{2}}, k_{c}, k_{s}\right)$ (where $g$ is a known function).

Equation (4) defines the relationship among the unconstrained swelling of the jellyroll $\left(s_{a}^{f}\right)$, the swelling of the case at constrained conditions $\left(s_{c}^{c}\right)$, and the initial displacement of the spacer $\left(s_{0}\right)$. Using this equation, the swelling of the case at constrained conditions can be derived in terms of the unconstrained swelling of the jellyroll and the initial displacement of the spacer as $s_{c}^{c}=h\left(s_{a}^{f}, s_{0} ; k_{a_{1}}, k_{a_{2}}, k_{c}, k_{s}\right)$ (where $h$ is a known function).

Finally, Eq. (5) can be expressed in terms of the measured swelling of the case at free conditions $\left(s_{c}^{f}\right)$ and the initial displacement of the case due to the preload $\left(s_{0_{c}}\right)$ by using $f, g$, and $h$ into Eq. (5) to obtain

$$
\begin{aligned}
& F_{m}=k_{s} \cdot h\left(f\left(s_{c}^{f} ; k_{a_{1}}, k_{a_{2}}, k_{c}\right), g\left(s_{0_{c}} ; k_{a_{1}}, k_{a_{2}}, k_{c}, k_{s}\right) ; k_{a_{1}}, k_{a_{2}}, k_{c}, k_{s}\right) \\
& +\left(k_{s}+k_{c}+k_{a_{1}}\right) s_{0_{c}}+k_{a_{2}} s_{0_{c}}^{3} .
\end{aligned}
$$

The full expression of Eq. (6) is omitted here for the sake of brevity. The inputs are the free swelling of the case at free conditions and the initial displacement of the case, suggesting that the force due to Li-ion intercalation at actual pack conditions can be estimated if the free swelling in terms of SOC and initial displacement are available.

\subsection{Parameterization and validation}


Figure 3 (a) illustrates the measured force and swelling over SOC due to Li-ion intercalation during discharge at $25^{\circ} \mathrm{C}$. The solid line, dotted line, and dash-dotted line are the quasi-static force, quasi-static swelling, and dynamic (transient) swelling at the center point on the battery cell (labeled as 13 in Figure 1). The overall trends for the force and swelling are similar, suggesting that these trends have the same physical origin. The volumetric changes in each electrode cannot be distinguished from the available measured force and swelling. However, the volumetric changes are due mainly to the volume change of the negative electrode (made of graphite intercalation material) because the volume change of the positive electrode (made of nickel/manganese/cobalt-oxide) is less than $1 \%$ [29]. Moreover, the overall shape of the measured swelling and force is similar to the average interlayer spacing of graphite within the negative electrode [30]. These observations support the hypothesis that the measured force and swelling can be attributed mainly to volumetric changes within the negative electrode due to Liion intercalation. Note that the measured force is around $600 \mathrm{~N}$ at $0.0 \mathrm{SOC}$ because of the preload. The difference between the quasi-static swelling and the transient swelling measured at $0.2 \mathrm{C}$ is almost the same in high and low SOC regions. In contrast, the difference around $0.5 \mathrm{SOC}$ is larger than other regions even though it is not significant; the maximum difference is $4.5 \mu \mathrm{m}$ in the middle range of SOC. This observation coincides well with the dependency of phase volume fraction on the C-rate [31] and the surface structural disordering during transient states [32]. Especially, the volume fraction of $\mathrm{Li}_{\mathrm{x}} \mathrm{C}_{6}$ depends significantly on the C-rate [31]. Thus, the swelling in the middle range of SOC might depend on the C-rate. In contrast, negative electrodes have single phase in high and low SOC regions, suggesting that the swelling might be constant regardless of the $\mathrm{C}$-rate in these regions. 
The effects of phase transition are more clearly observed in the measured force in terms of swelling at steady-state (shown as symbols in Figure 3 (b)). The force increases nonlinearly over the SOC and displays a piece-wise parabolic shape in regions I ( $0 \%-40 \%$ SOC) and III $(65 \%$ $100 \%$ SOC). Moreover, a sharp change in the relationship between force and swelling is observed in the middle range of SOC, region II $(40 \%-65 \%)$. This observation suggests that material properties depend on the SOC due to some of the phase transitions [33]. Based on these experimental observations, we hypothesize that material properties do not change significantly in region I and region III. Thus, the equivalent stiffness of a jellyroll is constant in region I and separately in region III, but these values are different because of phase differences.

The small variation in material properties in regions can be explained by the micro-structure. Denote by stage-n and stage-nL a single Li intercalated layer for every $\mathrm{n}$ graphite sheets, where L indicates the disordered (liquid-like) lithium-ion intercalation (see Ref. [33] for the details about the phase transition of the graphite intercalation). A region is referred to as stage-n when stage-n is dominant compared to other phases in that region, but stage-n not the only stage.

The material properties of stage- $4 \mathrm{~L}$, stage- $3 \mathrm{~L}$, and stage- $2 \mathrm{~L}$ are assumed to be similar for low SOC (region I) and material properties of stage- 2 and stage- 1 are assumed to be similar for high SOC (region III). Transitions between stages can lead to a mixture of several stages which can merge and separate during transitions [50].

Note also that the change in porosity in the separator might also contribute to nonlinearity in high and low SOC regions. A decrease in porosity can result in a higher stiffness of the jellyroll because more material is bearing the load [35], manifesting itself as an upward slope in Figure 3 (b). 
Material properties significantly change in the middle range of SOC (region II). Two possible reasons are that multiple phases exist around $0.5 \mathrm{SOC}[31,35]$, that the liquid-like stage (stage2L) changes to the solid stage (stage-2) [33], or combination thereof. Although, the exact origin of this significant change in the middle range of SOC is not understood completely, experimental results show that the equivalent stiffness of the jellyroll for region II is a combination of that for regions I and III. Therefore, $k_{a_{1}}$ and $k_{a_{2}}$ for region II were estimated by using

$$
k_{a(R-I I)}=\alpha k_{a(R-I)}+\beta k_{a(R-I I)}(\text { where } \alpha+\beta=1),
$$

where $\alpha$ and $\beta$ denote contribution factors for each phase respectively, and $R-\bullet$ in the subscript brackets denotes the region. The stiffness of the spacer $k_{s}$, that of the case $k_{c}$ as well as the initial displacement of the battery cell $s_{0_{c}}$ (due to preload) are the same for all regions because the phase transition does not affect these parameters.

In summary, seven parameters, namely $k_{s}, k_{c}, k_{a_{1}(R-I)}, k_{a_{2}(R-I)}, k_{a_{1}(R-I I)}, k_{a_{2}(R-I I I)}$, and $s_{0_{c}}$, were estimated from the measured force and swelling with a nonlinear least squares method. Then, $\alpha$ and $\beta$ were explicitly calculated with estimated parameters and the force to estimate the equivalent stiffness of the jellyroll for region II.

The solid line in Figure 3 (b) shows the nonlinear least square fitting results with identified parameters based on measurements (triangle symbols). The coefficient of determination $\left(R^{2}\right)$ is over 0.996 for all fitted regions, suggesting that the fitted curves are consistent with measured data. The identified parameters are listed in Table 1. The equivalent stiffness for the case is much smaller than that of the jellyroll because the case is very thin. However, the equivalent stiffness 
of the jellyroll is much smaller than that of the spacer. This may be due to the voids between in the rolled jellyroll and/or due to the electrolyte inside of the battery cell [34].

Table 1. Estimates of the equivalent stiffness in the model which relates force and swelling over SOC measured by displacement sensors and load cells.

\begin{tabular}{|c|c|c|}
\hline Parameter & Value & Unit \\
\hline$k_{s}$ & $3.30 \times 10^{8}$ & $\mathrm{Nm}^{-1}$ \\
\hline$k_{c}$ & $1.42 \times 10^{5}$ & $\mathrm{Nm}^{-1}$ \\
\hline$k_{a_{1}(R-I)}$ & $3.31 \times 10^{6}$ & $\mathrm{Nm}^{-1}$ \\
\hline$k_{a_{2}(R-I)}$ & $6.80 \times 10^{13}$ & $\mathrm{Nm}^{-3}$ \\
\hline$k_{a_{1}(R-I I I)}$ & $4.45 \times 10^{6}$ & $\mathrm{Nm}^{-1}$ \\
\hline$k_{a_{2}(R-I I I)}$ & $5.40 \times 10^{13}$ & $\mathrm{Nm}^{-3}$ \\
\hline
\end{tabular}

For validation, the model predictions were compared to measurements at different preloads. The initial displacement $\left(s_{0_{c}}\right)$ was the only value adjusted to match the initial force at $0.0 \mathrm{SOC}$ with the same equivalent stiffness of each component as listed in Table 1 . The dashed line and the dotted line show model predictions in Figure $3(\mathrm{~b})$, whereas the square symbols and the circle symbols show measured data at different preloads. The model predictions are in excellent agreement with the measured data, suggesting that the estimated parameters are reliable and accurate and thereby the proposed model can predict the electro-chemical induced force at any preload during the operation conditions, i.e. actual pack conditions.

Figure 3 (c) illustrates the variation of the contribution factors $\alpha$ and $\beta$ over SOC. A solid line shows values of $\alpha$, while a dashed line shows values of $\beta$. The constant values of $\alpha$ and 
$\beta$ are shown for regions I and III also. In these regions, $\alpha$ and $\beta$ are either zero or unity. In contrast, the value of $\alpha$ decreases and the value of $\beta$ increases over SOC in region II as we assume that the equivalent stiffness of the jellyroll for region II depends on the phase transitions occurring in that region. Figure 3 (d) shows the variation of the equivalent stiffness over SOC. Similar to the contribution factors, each equivalent stiffness is constant in regions I and III, whereas they vary in terms of SOC in region II.

\section{Phenomenological relaxation model}

The key idea for a relaxation model is that the force due to the volume change of a cell has two main origins: elasticity and $1^{\text {st }}$ order viscoelastic mechanical relaxation. However, no viscous force occurs at steady state where all velocities are zero. One obtains

$$
\begin{gathered}
F_{E_{S}}=F_{E_{D}}+F_{V_{D}} \\
=F_{E_{D}}+c \dot{s}_{c_{D}}^{f},
\end{gathered}
$$

where $F_{E_{S}}, F_{E_{D}}$, and $F_{V_{D}}$ represent the elastic force at steady state, the elastic force at a dynamic state, and the viscous force (at a dynamic state). $c$ and $\dot{s}_{c_{D}}^{f}$ denote viscosity and the velocity of the case at free condition (and a dynamic state). Thus, the swelling difference between the steady and dynamic states in high and low SOC regions may originate from the $1^{\text {st }}$ order viscoelastic mechanical relaxation as aforementioned in section 3. Note that the difference in the middle range of SOC is due to the dependency of phase volume fraction on the C-rate as well as the mechanical relaxation. 
Consider the relaxation of the battery cell following a full discharge. Since phase transitions have concluded before this relaxation period starts, the temporal variation of the force and swelling can be modeled with two $1^{\text {st }}$ order relaxations (two time constants); one for the thermal relaxation and the other for the viscoelastic mechanical relaxation [26].

The elastic force in a battery cell due to Li-ion intercalation is the sum of the force due to two spring elements in Figure 2 (a) at free swelling conditions (because these two springs are connected in parallel), namely $F_{E}=\left(k_{s}+k_{a_{1}}\right) s_{c}^{f}+k_{a_{2}}\left(s_{c}^{f}\right)^{3}$. Therefore, the elastic force at steady and also at dynamic states can be estimated with the measured swelling at steady $s_{c_{s}}^{f}$ and dynamic states $s_{c_{D}}^{f}$. Using Eq. (8), the balance of forces can be expressed as

$$
c_{0} \dot{s}_{c_{D}}^{f}(t)=\left(\left(k_{s}+k_{a_{1}}\right) s_{c_{S}}^{f}(t)+k_{a_{2}} s_{c_{S}}^{f 3}(t)\right)-\left(\left(k_{s}+k_{a_{1}}\right) s_{c_{D}}^{f}(t)+k_{a_{2}} s_{c_{D}}^{f 3}(t)\right)
$$

where $c_{0}$ is an equivalent coefficient of the $1^{\text {st }}$ order viscoelastic relaxation at 0.0 SOC. The coefficient $c_{0}$ can be estimated by using measurements at one SOC value (e.g., at 0.0 SOC) to obtain

$$
c_{0}=\frac{1}{\dot{s}_{c_{D}}^{f}(t)}\left[\left(\left(k_{s}+k_{a_{1}}\right) s_{c_{S}}^{f}(t)+k_{a_{2}} s_{c_{S}}^{f^{3}}(t)\right)-\left(\left(k_{s}+k_{a_{1}}\right) s_{c_{D}}^{f}(t)+k_{a_{2}} s_{c_{D}}^{f^{3}}(t)\right)\right],
$$

The results in Figure 3 show that there is a difference between the measured quasi-static swelling and dynamic swelling following a $0.2 \mathrm{C}$-rate discharge. In particular, this difference can be observed at $0.0 \mathrm{SOC}$ after the current has been stopped. The value of $\dot{s}_{c_{D}}^{f}$ can be obtained by using finite differences in time from the measured data. Next, the value of $\dot{s}_{c_{D}}^{f}$ together with the 
values of the quasi-static and the dynamic swelling at the moment when the SOC is 0.0 can be plugged into Eq. (10)) to obtain $c_{0}$. The estimated $c_{0}$ is $0.008 \mathrm{Nsm}^{-1}$.

Note that the stiffness coefficients and the coefficient of the $1^{\text {st }}$ order viscoelastic relaxation in Eqs. (9)) and (10)) depend on the SOC. Hence, we use Eqs. (9)) and (10)) only for a fixed value of SOC, namely 0.0 .

The proposed approach was applied to the relaxation period at the end of a full discharge. The initial swelling for the relaxation period was the swelling measured at the end of a $0.2 \mathrm{C}$ discharge. The equivalent stiffness values used were those obtained for region I (see Table 1) because the $\mathrm{SOC}$ is 0.0 during this relaxation.

Figure 4 compares the measured dynamic swelling (shown by the symbols) and the model prediction (shown by the solid line) during a three hour relaxation period. The model prediction is in great agreement with the measured dynamic swelling considering the accuracy of the displacement sensor $(1 \mu \mathrm{m})$. The maximum difference between the model and measure swelling is $0.75 \mu \mathrm{m}$, and the root-mean-square (RMS) difference is $0.70 \mu \mathrm{m}$. This suggests that the estimated parameters are accurate and reliable.

Please note the assumptions employed for this model. These require careful consideration when applying this model. Also, the proposed method is an empirical approach whose use requires verification at more SOC values. Moreover, the model requires extensions and modifications for using it in the multi-phase region, i.e. around 0.5 SOC.

\section{Phenomenological 3-D swelling model}




\subsection{Model description}

To predict the swelling shape on the surface during various operational conditions, a 3-D numerical and phenomenological swelling model is proposed in this section. The size and location of the jellyroll inside a battery cell was estimated from the X-ray tomography (shown as a yellow domain in Figure 5) [37]. Information from the dissection showed that the active material region is only slightly larger than the overall contact region between the jellyroll and the battery case. Hence, the contact region was approximated as the active material region (shown as a purple domain in Figure 5). To distinguish the contact region (the center region of the jellyroll) from the noncontact region (top and bottom of the jellyroll), a $100 \mu \mathrm{m}$ gap is modeled between the case and the jellyroll at the top and bottom sides of the jellyroll in the finite element model. Also, only the active material which contacts the case can contribute to the swelling of the case.

The models for other components such as the Aluminum battery case (shown as a semitransparent gray domain in Figure 5) and the plastic spacer (shown as a semi-transparent blue domain in Figure 5), which was made from polybutylene terephthalate by using injection mold, were also created with measured dimensions. The modulus of elasticity of the Aluminum case is $68 \mathrm{GPa}$. The modulus of elasticity of the spacer is $3.6 \mathrm{GPa}$, a value measured by nanoindentation and found to be consistent with previously reported values for polybutylene terephthalate.

These models were created to simulate two conditions. First, the free swelling of the battery cell was simulated to depict the measured swelling shape on the surface of the battery cell; two components, namely the case and the jellyroll, were used for this analysis. Second, the constrained swelling was simulated to illustrate actual pack conditions [28]; four components, 
namely one case, one jellyroll, and two half spacers, were used. All components were modeled as a homogeneous solid media.

The jellyroll was modeled as a cuboid similar to Ref. [40]. The wound shape of jellyroll was not considered in that this part does not contact the case, and thereby it does not contribute to the swelling of the case. Only half of the plastic spacer was modeled on each side of the battery cell to reduce computational time. The spacer model was assembled with the battery cell model by using appropriate boundary conditions. For example, no separation with slip was assigned between the jellyroll and the battery case, between the battery case and the spacer, and between the spacer and the exterior walls. The resulting overall model simulates one of the three cells and the corresponding spacers used in the force experiments. Other components such as bus bars and terminals were not modeled for simplicity. It is assumed that the effects of these un-modeled components are relatively small and local, and thereby negligible.

\subsection{Estimation of equivalent material properties}

Simulations were carried out with a structural analysis package of ANSYS. An orthotropic thermal expansion was induced in the jellyroll to illustrate the Li-ion intercalation swelling because the swelling due to Li-ion intercalation is not directly available in the analysis package

of ANSYS. The constitutive equation of the mechanical (elastic) strain with thermal strain and diffusion induced strain can be expressed as follows [19]

$$
\varepsilon_{i j}=\frac{1}{E}\left((1+v) \sigma_{i j}-v \sigma_{k k} \delta_{i j}\right)+\frac{1}{3} \Omega \Delta c \delta_{i j},
$$




$$
\varepsilon_{i j}=\frac{1}{E}\left((1+v) \sigma_{i j}-v \sigma_{k k} \delta_{i j}\right)+\alpha_{i j} \Delta T \delta_{i j}
$$

where $\varepsilon, E, v, \sigma$, and $\delta$ denote the strain, the modulus of elasticity, Poisson's ratio, the stress, and the Kronecker delta respectively. The coefficient of thermal expansion and the temperature variation from the reference value are denoted by $\alpha$ and $\Delta T . \Omega$ and $\Delta c$ are partial molar volume representing volume expansion caused by concentration of solute atoms, and the concentration variation of diffusion species from the reference value [19].

Equations (11) and (12) show that the intercalation-induced strain/stress has an analogy to an anisotropic thermal strain/stress. Stresses caused by diffusion of solute atoms lead to a stressstrain relation similar to that due to temperature gradients. Also, the coefficient $\Omega / 3$ in Eq. (11) plays a role similar to the coefficient of thermal expansion in the analysis of thermal stress [41,42]. This analogy suggests that computational solvers designed for modeling thermal expansion can be used for analyzing Li-ion intercalation expansion. This approach was used already to estimate the stress and strain due to Li-ion intercalation in graphite electrodes $[24,43]$. Consider a Li-ion intercalation swelling in the $z$ direction. An equivalent thermal expansion can be defined in the $z$ direction while a small value of 0.01 of Poisson's ratio can be used to minimize the relative expansion in the $x$ and $y$ directions due to the swelling in the $z$ direction. This approach accounts for the orthotropic expansion of Li-ion intercalation in a direction perpendicular to the electrodes. 
Estimating an equivalent modulus of elasticity to be used in the 3-D model for the jellyroll is more complex. Consider a battery cell constrained with no preload between two rigid walls (plates) via spacers (as shown in Figure 7). A force $F$ is generated on the constraining walls when the active material inside the battery cell swells. For a large stiffness of the spacer and of the case, the deformations of the spacer and the case are negligible because they are contacting the fixed walls. Hence, the case and the spacer do not contribute significantly to the force $F$. The active material is the component which contributes to $F$.

Now consider the swelling of the active material when it is completely free, unconstrained by the case, spacers or walls. For the same electrochemical and thermal states, the swelling of the active material is $s_{a}^{f}$ (as depicted in Figure 2). The ratio between $F$ and $h$ is an equivalent stiffness of the active material $k_{a_{e q}}$. This value is proportional to the modulus of elasticity $E_{a_{e q}}$ of the active material. This modulus of elasticity depends on the SOC because $k_{a_{e q}}$ depends on the SOC.

The coefficient of proportionality between the modulus and the equivalent stiffness in the 3-D model has to be the same as in the 1-D model. Thus, when one can use an arbitrary (non-zero, non-infinite) modulus of elasticity $E_{\text {ANSYS }}$ in the 3-D model in ANSYS, consider a swelling of the material and calculate an equivalent stiffness $k_{A N S Y S}$. Then, the ratio $r=\frac{E_{A N S Y S}}{k_{A N S Y S}}$ does not depend on the arbitrary selection of $E_{A N S Y S}$. The value $r$ is a constant characteristic of the 3-D shape of the battery cell. It does not depend on the SOC and is easily determined by a single ANSYS static calculation of constrained swelling modeled as orthotropic thermal expansion and a small value of Poisson's ratio as discussed above.

Since the ratio $r$ is the same in the 3-D and 1-D models, one obtains 


$$
\frac{E_{a_{e q}}(S O C)}{k_{a_{e q}}(S O C)}=\frac{E_{A N S Y S}}{k_{A N S Y S}}=r .
$$

The value of $k_{a_{e q}}$ in Eq. (13) is available from the 1-D model obtained through measurements. Specifically, Eq. (5) from the 1-D model provides the value of $F$ for a constrained swelling with zero pre-stress (and hence an approximately zero deformation of the case and spacer, $s_{c}^{c}=0$ ) as

$$
F=k_{a_{1}} s_{a}^{f}+k_{a_{2}}\left(s_{a}^{f}\right)^{3}, \text { and hence } k_{a_{e q}}=\frac{F}{s_{a}^{f}}=k_{a_{1}}+k_{a_{2}}\left(s_{a}^{f}\right)^{2}
$$

where $k_{a_{1}}$ and $k_{a_{2}}$ depend on the SOC as discussed in section 3. Next, one obtains the equivalent modulus of elasticity of the active material using Eq. (14) into Eq. (13) as follows

$$
E_{a_{e q}}(S O C)=r k_{a_{e q}}(S O C)=r\left[k_{a_{1}}(S O C)+k_{a_{2}}\left(s_{a}^{f}(S O C)\right)^{2}\right]
$$

Figure 6 illustrates the evolution of equivalent modulus of elasticity over SOC. The solid, dashed, and dotted lines denote the equivalent modulus of elasticity for the jellyroll $E_{a_{e q}}$, its linear part (due to $k_{a_{1}}$ ), and its nonlinear part (due to $k_{a_{2}}$ ). These results show that the linear term is constant in the low and high SOC regions. As expected, the linear term changes around 0.5 SOC due to phase transition, and the nonlinear term monotonically increases over SOC. These observations are a direct consequence of the results in Figure 5 (b). The nonlinear term contributes over $20 \%$ of the total modulus of elasticity at the fully charged state, suggesting that it is not negligible. The equivalent modulus of elasticity for the jellyroll $E_{a_{e q}}$ also varies nonlinearly over SOC and shows a sudden change due to phase transition. However, the estimated equivalent modulus of elasticity for the jellyroll is much smaller than that of other battery components such as a cathode, an anode, and even a separator [45-47]. These results 
could be explained by the combined effect of the separator and electrolyte. Hundreds of ultrathin film electrodes are stacked in the jellyroll. The lowest stiffness of all these layers influences the most the equivalent stiffness of the entire system (like in a series connection of springs). Hence, the stiffness of the separator might be dominant. Moreover, electrolyte fills the pores of the separator. Hence, the stiffness (and hence the equivalent modulus) of the separator can be represented as a combination of two spring elements (separator, electrolyte) connected in series for some regions and in parallel for other regions. Consequently, the equivalent modulus of elasticity for the cell can be of the order of one MPa because the modulus of the electrolyte is around $1 \mathrm{MPa}[19,48]$, while that of the separator is of the order of hundreds of MPa [44]. However, the exact physical origin of the measured values is still not completely clear and further investigations are needed to elucidate the physical basis for the observed values.

\subsection{Model validation}

The measured 2-D swelling shape on the surface of the battery cell at the fully charged state (the third experiment in section 2) is shown in Figure 7 (a). The swelling is almost flat in the central area because of the flat wound jellyroll in that area and because Li-ion intercalation is uniform in the $x y$ plane (the plane of the surface of the battery). In contrast, the swelling near the sides varies and is relatively small due to constraints from the case and due to the empty space inside the case, as shown in the X-ray tomography [37]. Relatively large expansion is observed at the bottom right side on the surface. That can be caused by the bus bars and the clamps which are inside the case and bind the jellyroll at its left side (also visible in the X-ray tomography). As described in detail in Ref. [26], the complex shape of the jellyroll and the empty space inside the 
case result in asymmetric swelling at the edges. However, the effects of these complex components are not extending in the center of the cell where swelling is uniform. Therefore, the best locations for monitoring free swelling with just few sensors are in the center region.

Figure 7 (b) shows the free swelling shape on the surface of the battery case from the 3-D phenomenological model at the fully charged state (1.0 SOC). The contour plot uses the same contour line values as those in Figure 7 (a) for easy comparison. In simulation, the orthogonal expansion in a direction perpendicular to the electrode is induced to match the magnitude of the swelling at the center of the surface (labeled as point 13 in Figure 1). Then, the model predictions are compared with measurements of swelling at other locations. The overall swelling shape and the magnitude of swelling are similar to experiments, except for the effect of the unmodeled clamps and bus bars.

Figure 7 (c) shows the swelling along horizontal lines H1 to H5 labeled in Figure 1. Solid lines show estimated swelling from the 3-D model, while symbols show measured swelling at the fully charged state. The model prediction at the center region (H2 to H4) is in agreement with measured data. The swelling at the top side (H1) is also consistent with the experiments. However, the bottom side (H5) shows differences between the model and the measured data. This might be the effect of the asymmetrically located clamps and bus bars as aforementioned. The comparison between model prediction and experiments suggests that the assumptions made in constructing the model are reasonable, namely the wound shape of the jellyroll does not contribute significantly to the swelling shape of the case (due to empty spaces), and the bus bars and clamps do not need to be modeled for evaluating the shape of swelling, especially in the center regions. 
Figure 7 (d) illustrates the quasi-static free swelling at a variety of SOC (the second experiment). Three horizontal lines (labeled as H2, H3, and H4 in Figure 1) show the model predictions, whereas symbols show measured data. Solid, dashed, and dotted lines denote free swelling at 0.9 , 0.5 , and 0.3 SOC respectively. The swelling shape predicted by the 3-D model is consistent with the measurements, suggesting that the orthotropic expansion model of the Li-ion intercalation swelling and the identified modulus of elasticity of the jellyroll are accurate. The free swelling predictions at other SOCs also are consistent with the measurements. Those results are omitted here for the sake of brevity. Simulations with the 3-D swelling model demonstrate that the proposed model is able to accurately predict the overall swelling shape on the surface of the cell.

\subsection{Simulation at pack conditions}

The proposed 3-D model for one battery cell is useful in several ways. For example, this model can be used to construct the model of a battery pack. Then, the force created in the pack by Liion intercalation can be estimated while also accounting for preload effects. Based on the battery cell or pack model, optimal displacement or strain sensor locations and the optimal number of sensors needed can be determined during design.

Another related example is related to predicting the constrained swelling shape on the surface of the battery cells in pack conditions at the fully charged state. This shape is shown in Figure 8 (a). Relatively large swelling can be observed in the center region between the areas of contact with the spacer. This result suggests that geometric constraints on the cell swelling due to spacers are important in pack conditions. Moreover, the pattern of swelling is repetitive and the amount of 
swelling (especially in the areas where there is no contact with the spacers) is the same in the center region, which is the area of contact between the jellyroll and the casing. Therefore, an appropriate location for placing the displacement or strain sensor in pack condition is the center region, in an area without contact with the spacer.

Figure 8 (b) shows the swelling predicted by the model over SOC in the center region, at a location where there is no contact with the spacer. The overall shape of expansion versus SOC is similar to the shape of the free swelling, indicating that innate characteristics of Li-ion intercalation swelling are observable also in constrained conditions. However, this swelling is an order of magnitude smaller, suggesting that high precision displacement or strain sensors are needed to use this information for estimating the SOC or the state of health (SOH).

The force due to Li-ion intercalation swelling in pack conditions was simulated (Figure 8 (c) and (d)). The main purpose of this simulation is to estimate the reaction force as a function of SOC between the spacer and the exterior walls, and comparing the estimated force from the 3-D model to that from the 1-D force model. The force from the 1-D model was estimated from Eq. (6) assuming that the initial displacement is zero. Hence, these simulation conditions correspond to the force due to Li-ion intercalation swelling without preload. This force also can be estimated from the finite element 3-D model. The variation of the jellyroll swelling due to the Li-ion intercalation (Figure 7 (d)) and its equivalent modulus of elasticity (Figure 6) over SOC were used as input parameters ranging from $0 \%$ to $100 \%$ SOC, with $10 \%$ increments. The reaction force between the spacers and the exterior walls was calculated with the 3-D model (Figure 5). Two half spacers as well as the case and the jellyroll are used in these simulations. 
Figure 8 (c) and (d) shows the reaction forces calculated from the 1-D force model and the 3-D model. The solid line is the force from the 1-D model, whereas the circle symbols are the force from the 3-D model. The forces from the 1-D model are consistent with the forces from the 3-D model. The maximum difference is $13 \mathrm{~N}$ and the root-mean-square (RMS) difference is $8 \mathrm{~N}$. These results corroborate the hypothesis that the force can be modeled with the nonlinear spring element reflecting the dependency on the magnitude of swelling (Eq. (6)), and the equivalent stiffness can be converted to an equivalent modulus of elasticity (Eq. (8)).

\section{Conclusions}

The mechanical properties, of either stack stress, strain, or a combination thereof, can provide inputs to SOC or SOH estimation algorisms and ultimately prolong the lifespan of the battery, not only of the cells but also of the packs. For these purposes, three phenomenological models are proposed based on the measured force and free swelling to depict the Li-ion intercalation behaviors. The 1-D models addresses the equivalent $1^{\text {st }}$ order relaxation or the nonlinear stiffness representing the force dependency on the magnitude of swelling, and thereby enables the proposed models to capture the transient swelling during the rest periods or steady-state force. The 3-D finite element model introduces the orthotropic expansion of the jellyroll and the equivalent modulus of elasticity to predict the swelling shape of the battery cell over SOC.

The 1-D models proposed herein are computationally effective and easily implementable for BMS for efficient control of battery cells and packs. These novel models are useful for electrified vehicles where unexpected exposure of the battery pack to severe environments can 
have dire consequences. For example, the fusing information from voltage, swelling, and force measurement enables BMS to estimate SOC more accurately and robustly, similar to Ref. [37]. The combination of the proposed models with electric-circuit models and thermal swelling models can predict the stresses due to Li-ion intercalation and that due to thermal swelling, and thereby facilitate more enhanced power strategies in BMS, similar to Ref. [28]. The framework can provide potential utility for the SOC or SOH estimation as a standalone algorithm or in hybrid forms with existing algorithms $[22,38,39]$ because swelling and its induced force are related to battery states and degradation. This work opens the door for an enhanced battery management system through the incorporation of mechanical measurements.

The proposed pack model also enables the creation of parametric reduced-order models for the fatigue life prediction or optimization of the HEVs [49], and thereby reduces the calculation time. The swelling shape can be extracted from the proposed model and compared to measured swelling data for enhanced power management or to facilitate SOC estimation and diagnostic algorithms because the swelling shape due to Li-ion intercalation is constant over SOC [26]. Ultimately, implementing in-situ monitoring of strain inside individual Li-ion battery cells with the proposed model in BMS holds great promise in improving the safety of a pack by allowing earlier detection of the onset of fracture or outgassing, which can lead to performance degradation of the cell.

\section{Acknowledgements}


The information, data, or work presented herein was funded in part by the Advanced Research Projects Agency-Energy (ARPA-E), U.S. Department of Energy, under Award Number DEAR0000269.

\section{Disclaimer}

The information, data, or work presented herein was funded in part by an agency of the United States Government. Neither the United States Government nor any agency thereof, nor any of their employees, makes any warranty, express or implied, or assumes any legal liability or responsibility for the accuracy, completeness, or usefulness of any information, apparatus, product, or process disclosed, or represents that its use would not infringe privately owned rights. Reference herein to any specific commercial product, process, or service by trade name, trademark, manufacturer, or otherwise does not necessarily constitute or imply its endorsement, recommendation, or favoring by the United States Government or any agency thereof. The views and opinions of authors expressed herein do not necessarily state or reflect those of the United States Government or any agency thereof.

\section{References}

[1] M. Bryner, Chemical Engineering Progress. 109 (10) (2013) 36-39.

[2] A. N. Jansen, Chemical Engineering Progress. 109 (10) (2013) 57-64.

[3] B. Scrosati, and J. Garche, Journal of Power Sources. 195 (2010) 2419-2430.

[4] L. Lu, X. Han, J. Li, J. Hua, and M. Ouyang, Journal of Power Sources. 226 (2013) 272-288.

[5] M. Chen, and G. A. Rinc'on-Mora, IEEE Transactions on Energy Conversion. 21 (2) (2006) 504-511.

[6] Nassim, Jason B. Siegel, and Anna G. Stefanopoulou, Proceedings of the ASME 2014 Dynamic Systems and Control Conference. San Antonio, USA (2014). 
[7] M. Park, X. Zhang, M. Chung, G. B. Lessa, and A. M. Sastry, Journal of Power Sources. 195 (2010) 7904-7929.

[8] S.-C. Chen, Y.-Y. Wang, and C.-C. Wan, Journal of the Electrochemical Society. 153 (4) (2006) A637-A648.

[9] S. Peck, T. Olszanski, S. Zanardelli, and M. Pierce, SAE Int. J. Passeng. Cars - Electron. Electr. Syst. 5 (1) (2012) 154-163.

[10] V. Srinivasan, and C. Y. Wang, Journal of the Electrochemical Society. 150 (1) (2003) A98A106

[11] Y. Chen, and J. W. Evans, Journal of the Electrochemical Society. 143 (9) (1996) 27082712.

[12] S. A. Khateeb, S. Amiruddin, M. Farid, J. R. Selman, and S. Al-Hallaj, Journal of Power Sources. 142 (2005) 345-353.

[13] Y. Kim, J. B. Siegel, and A. G. Stefanopoulou, 2013 American Control Conference. Washington, USA (2013).

[14] A. Santucci, A. Sorniotti, and C. Lekakou, Journal of Power Sources. 258 (2014) 395-407.

[15] V. A. Sethuraman, M. J. Chon, M. Shimshak, V. Srinivasana, and P. R. Guduru, Journal of Power Sources 195 (2010) 5062-5066.

[16] V.A. Sethuraman, N. V. Winkle, D.P. Abraham, A.F. Bower, and P.R. Guduru, Journal of Power Sources 206 (2012) 334-342.

[17] A. Mukhopadhyay, A. Tokranov, X. Xiaoc, and B. W. Sheldon, Electrochimica Acta. 66 (2012) 28-37.

[18] F. Kaasik, T. Tamm, M.M. Hantel, E. Perre, A. Aabloo, E. Lust, M.Z. Bazant, and V. Presser, Electrochemistry Communications. 34 (2013) 196-199.

[19] D. Shi, X. Xiao, X. Huang, and H. Kia, Journal of Power Sources. 196 (2011) 8129-8139.

[20] C. Peabody, and C. B. Arnold, Journal of Power Sources. 196 (2011) 8147-8153.

[21] J. Cannarella, and C. B. Arnold, Journal of Power Sources. 245 (2014) 745-751.

[22] J. Cannarella, and C. B. Arnold, Journal of Power Sources. 269 (2014) 7-14.

[23] J. H. Lee, H. M. Lee, S. Ahn, Journal of Power Sources. 119-121 (2003) 833-837.

[24] Y. Qi and S. J. Harris, Journal of the Electrochemical Society. 157 (6) (2010) A741-A747.

[25] J. B. Siegel, A. G. Stefanopoulou, P. Hagans, Y. Ding, and D. Gorsich, Journal of the Electrochemical Society. 160 (8) (2013) A1031-1038A.

[26] K.-Y. Oh, J. B. Siegel, L. Secondo, S. U. Kim, N. A. Samad, J. Qin, D. Anderson, K. Garikipati, A. Knobloch, B. I. Epureanu, C. W. Monroe, and A. Stefanopoulou, Journal of Power Sources. 267 (2014) 197-202.

[27] K.-Y. Oh, B. I. Epureanu, Journal of Power Source. under review.

[28] Y. Kim, S. Mohan, N. A. Samad, J. B. Siegel, and A. G. Stefanopoulou, 2014 American Control Conference, USA (2014)

[29] Y. Koyama, I. Tanaka, H. Adachi, Y. Makimura, and T. Ohzuku, Journal of Power Sources. 119-121 (2003) 644-648.

[30] R. Yazami, and Y. Reynier, Journal of Power Sources. 153 (2006) 312-318. 
[31] K. G. Gallagher, D. W. Dees, A. N. Jansen, D. P. Abraham, and S. H. Kang, Journal of the Electrochemical Society. 159 (2012) A2029-A2037.

[32] V. A. Sethuraman, L. J. Hardwick, V. Srinivasan, R. Kostecki, Journal of Power Sources. 195 (2010) 3655-3660.

[33] J. R. Dahn, Physical Review B. 44 (17) (1991) 9170-9177.

[34] N. Zhang, and H. Tang, Journal of Power Sources 218 (2012) 52-55.

[35] J. Cannarella, X. Liu, C. Z. Leng, P. D. Sinko, G. Y. Gor, and C. B. Arnold, Journal of the Electrochemical Society. 161 (11) (2014) F3117-F3122.

[36] K. D’Souza, B. I. Epureanu, Journal of Sound and Vibration. 329 (2010) 2463-2476.

[37] S. Mohan, Y. Kim, J. B. Siegel, N. A. Samad, and A. G. Stefanopoulou, Journal of the Electrochemical Society. 161 (14) (2014) A2222-A2231.

[38] G. K. Prasad, and C. D. Rahn, Journal of Power Sources. 232 (2013) 79-85.

[39] J. Schmalstieg, S. Käbitz, M. Ecker, and D. U. Sauer, Journal of Power Sources. 257 (2014) 325-334.

[40] U. Kim, P. Albertus, D. Cook, C. W. Monroe, and J. Christensen, Journal of Power Sources. 268 (2014) 625-633.

[41] F. Yang, Journal of Power Sources. 241 (2014) 146-149.

[42] X. Xiao, W. Wu, and X. Huang, Journal of Power Sources. 195 (2010) 7649-7660.

[43] M. Liu, Journal of Power Sources. 275 (2015) 760-768.

[44] I. C. Halalay, M. J. Lukitsch, M. P. Balogh, and C. A. Wong, Journal of Power Sources. 238 (2013) 469-477.

[45] X. Zhang, W. Shyy, and A. M. Sastry, Journal of the Electrochemical Society. 154 (10) A910-A916.

[46] K. R. Kganyago, and P. E. Ngoepe, Physical Review B 68 (2003) 205111.

[47] A. Sheidaei, X. Xiao, X. Huang, J. Hitt, Journal of Power Sources (2011) 8728-8734.

[48] R. E. Garcia, Y. M. Chiang, W. C. Carter, P. Limthongkul, and C. M, Bishop, Journal of the Electrochemical Society. 152 (2005) A255-A263.

[49] S.-K. Hong, B. I. Epureanu, M. P. Castanier, Journal of Power Sources. 261 (2014) 101-111. [50] G. Kirczenow, Physical Review Letters. 55 (25) (1985) 2810-2813.

[51] Y. Kim, N. A. Samad, K.-Y. Oh, J. B. Siegel, B. Epureanu, A. G. Stefanopoulou, ACC 2016. 


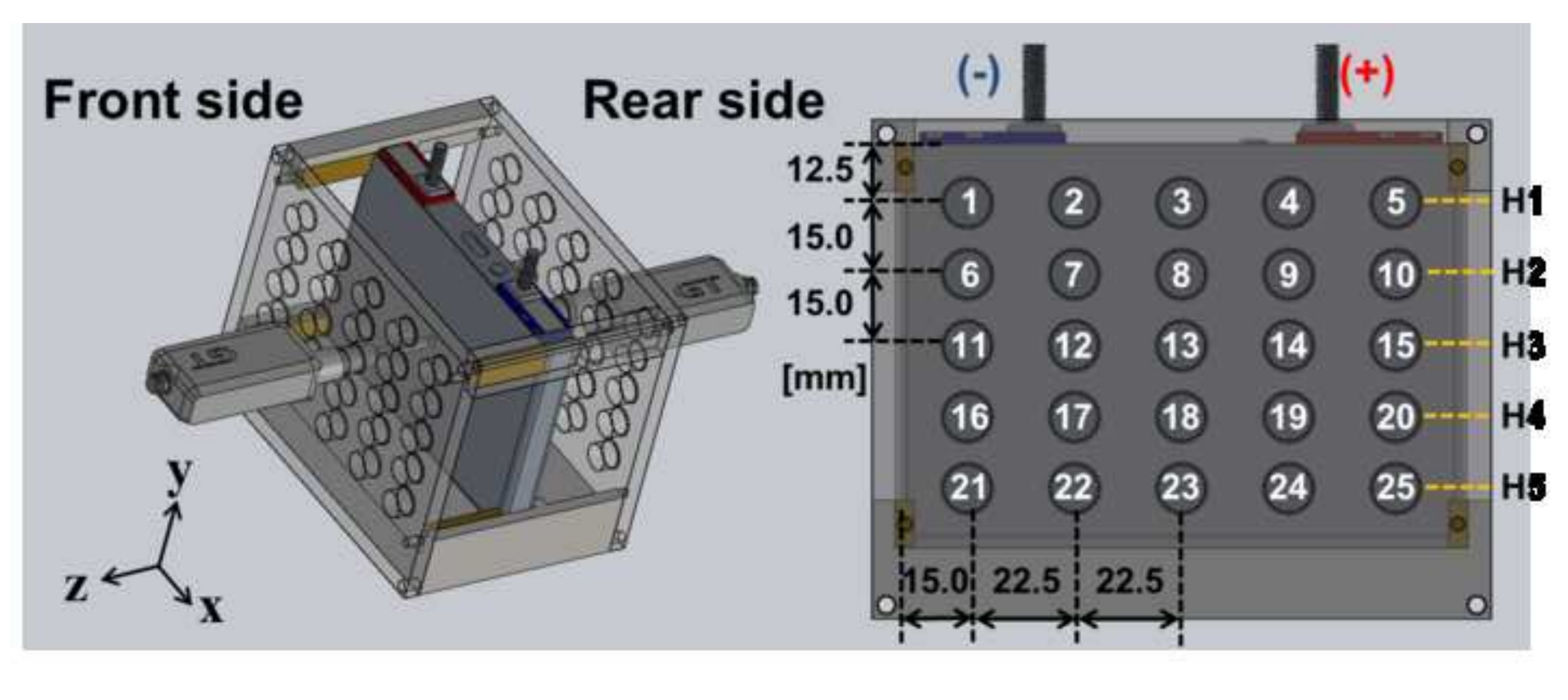

Front side
Rear side 
(a) Battery cell (free swelling)

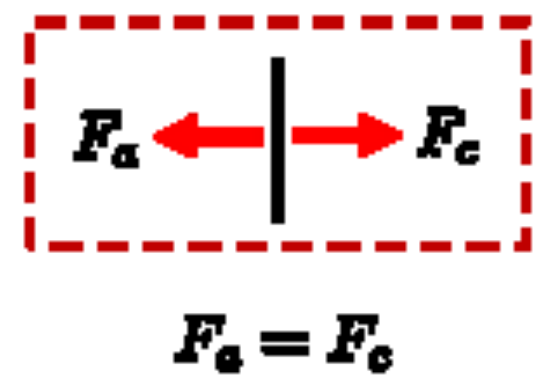

(b) Battery cell w/ spacer + preload

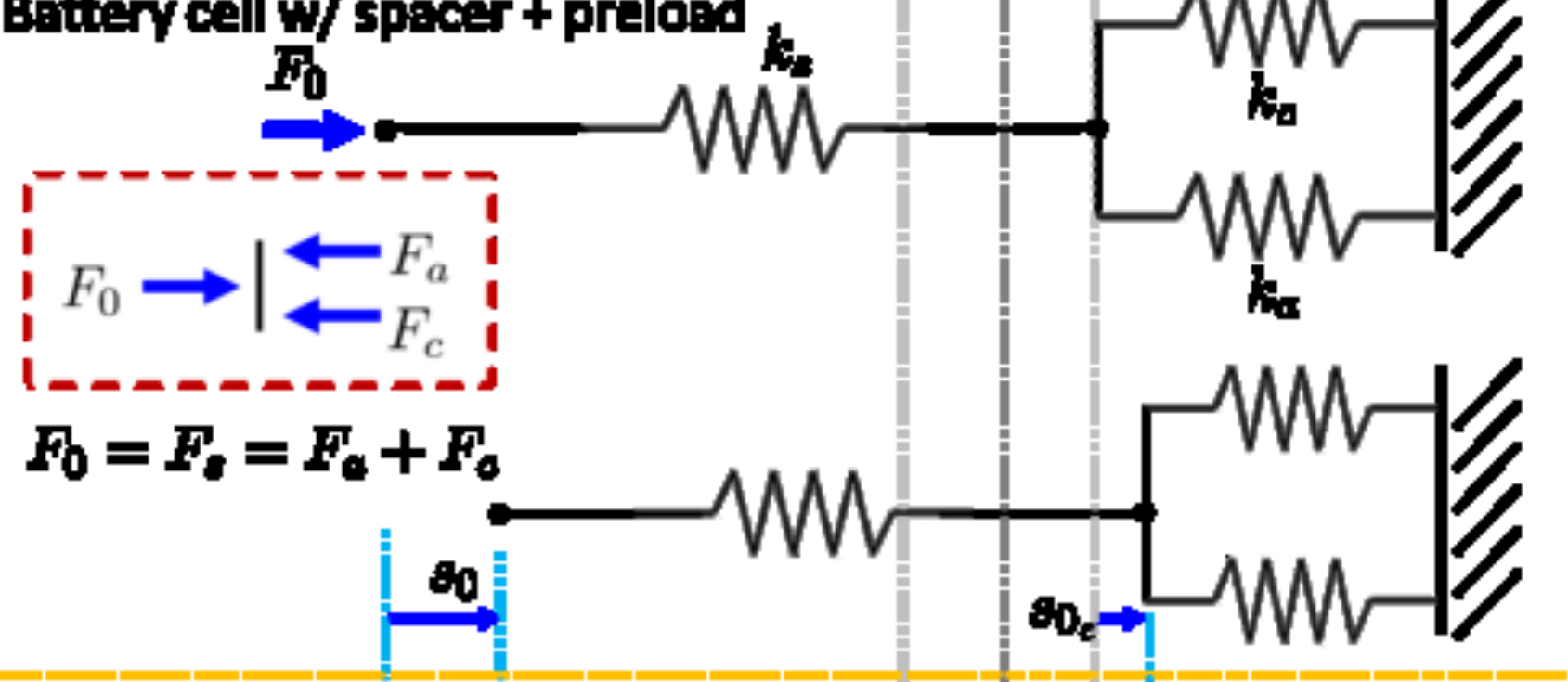

(c) Battery cell w/ spacer + preload + fix

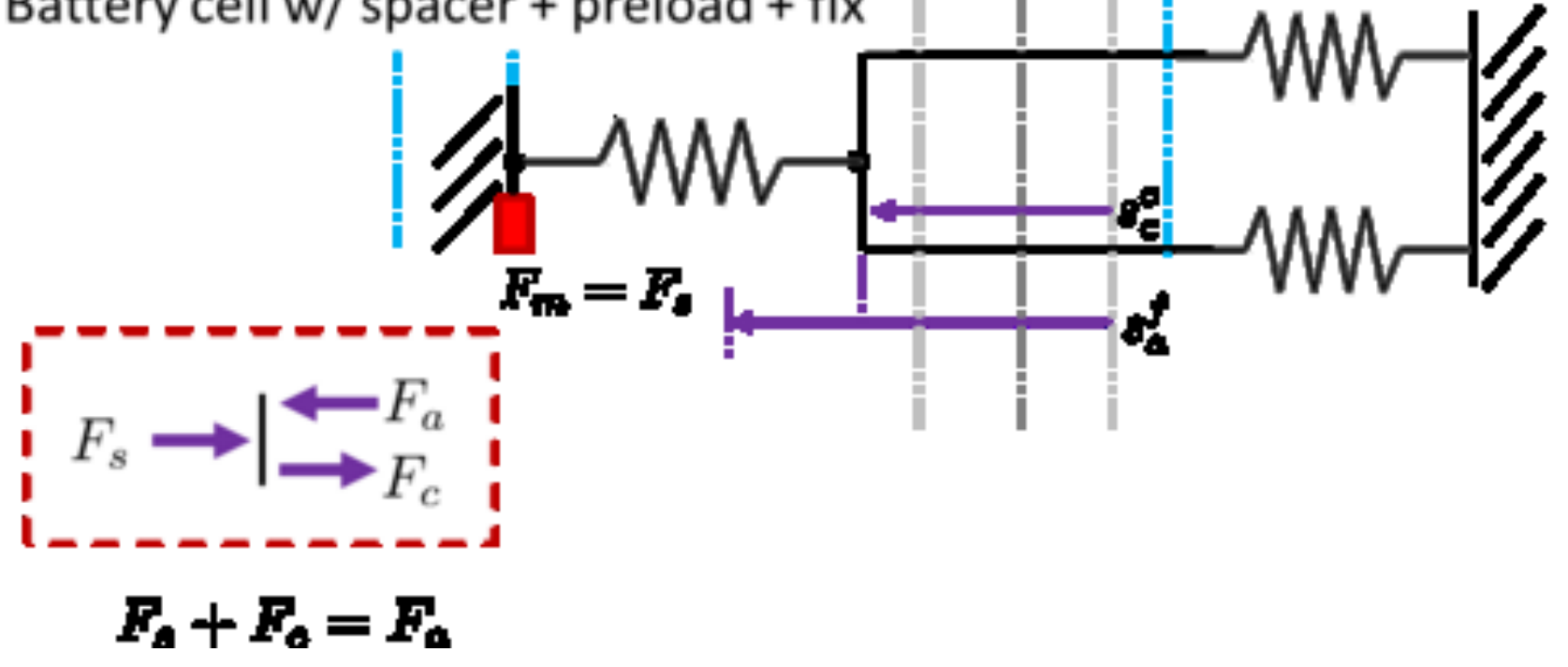


(a) 1800

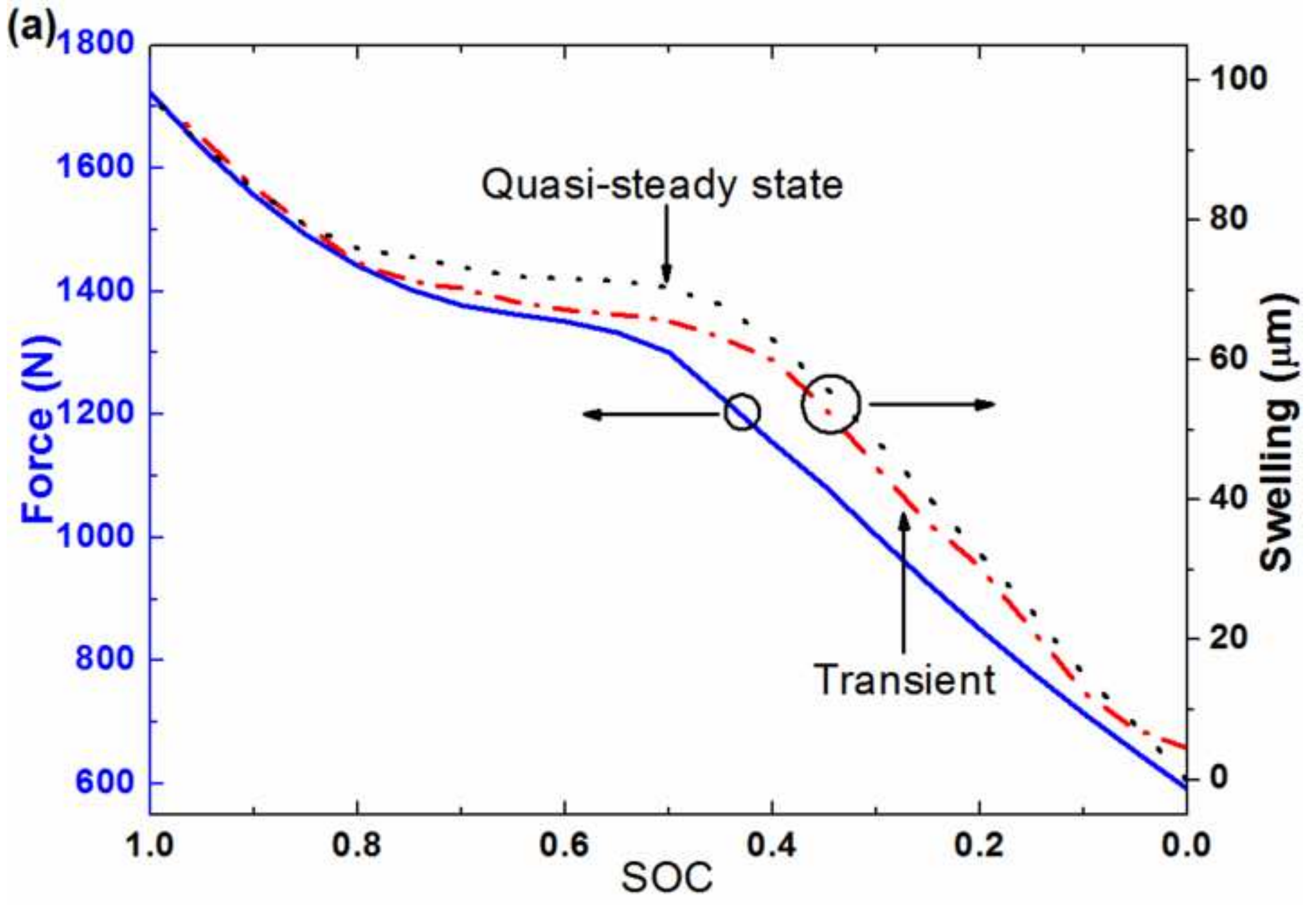


(b)

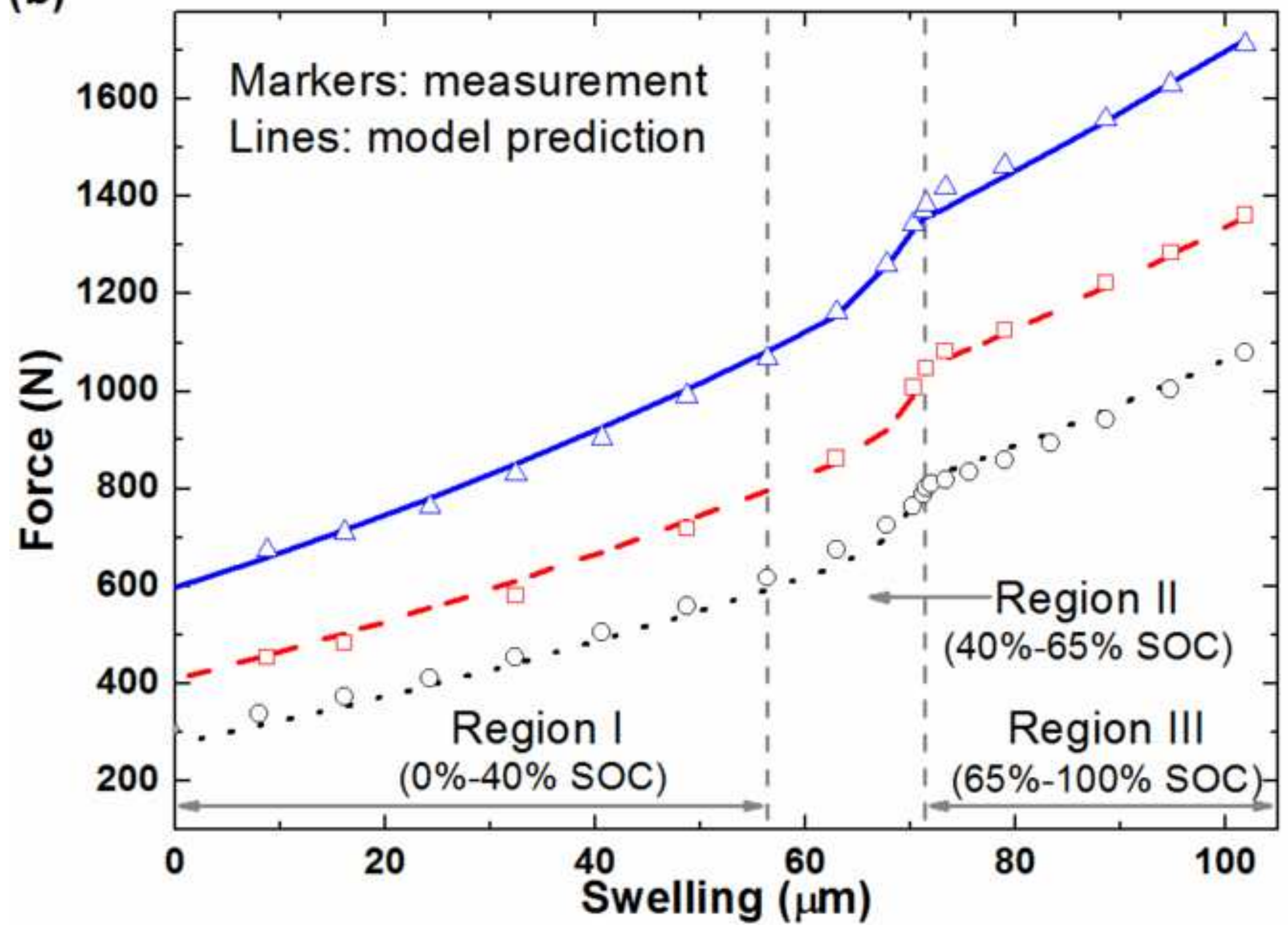


(c)

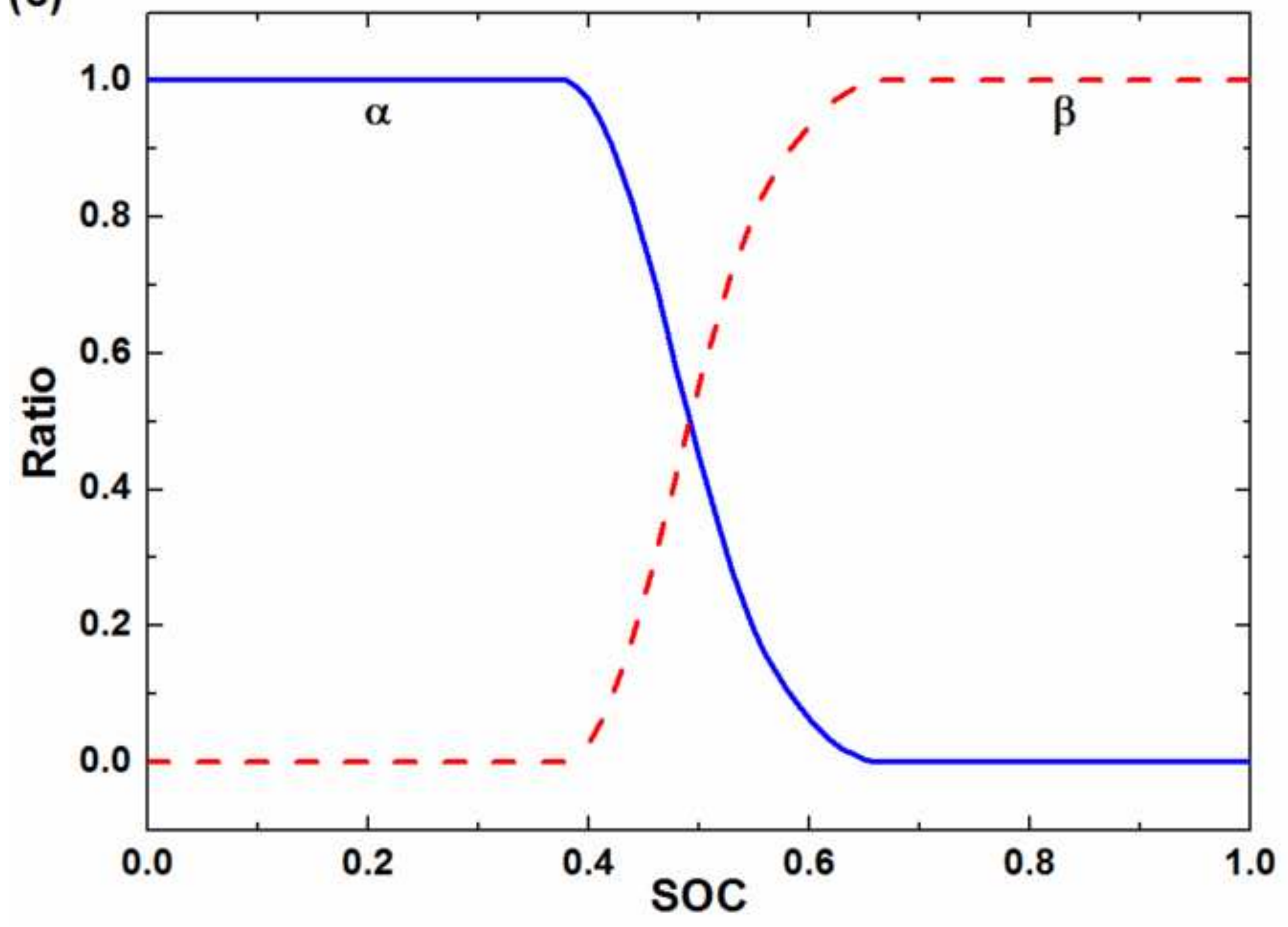

gure 3 (c) 


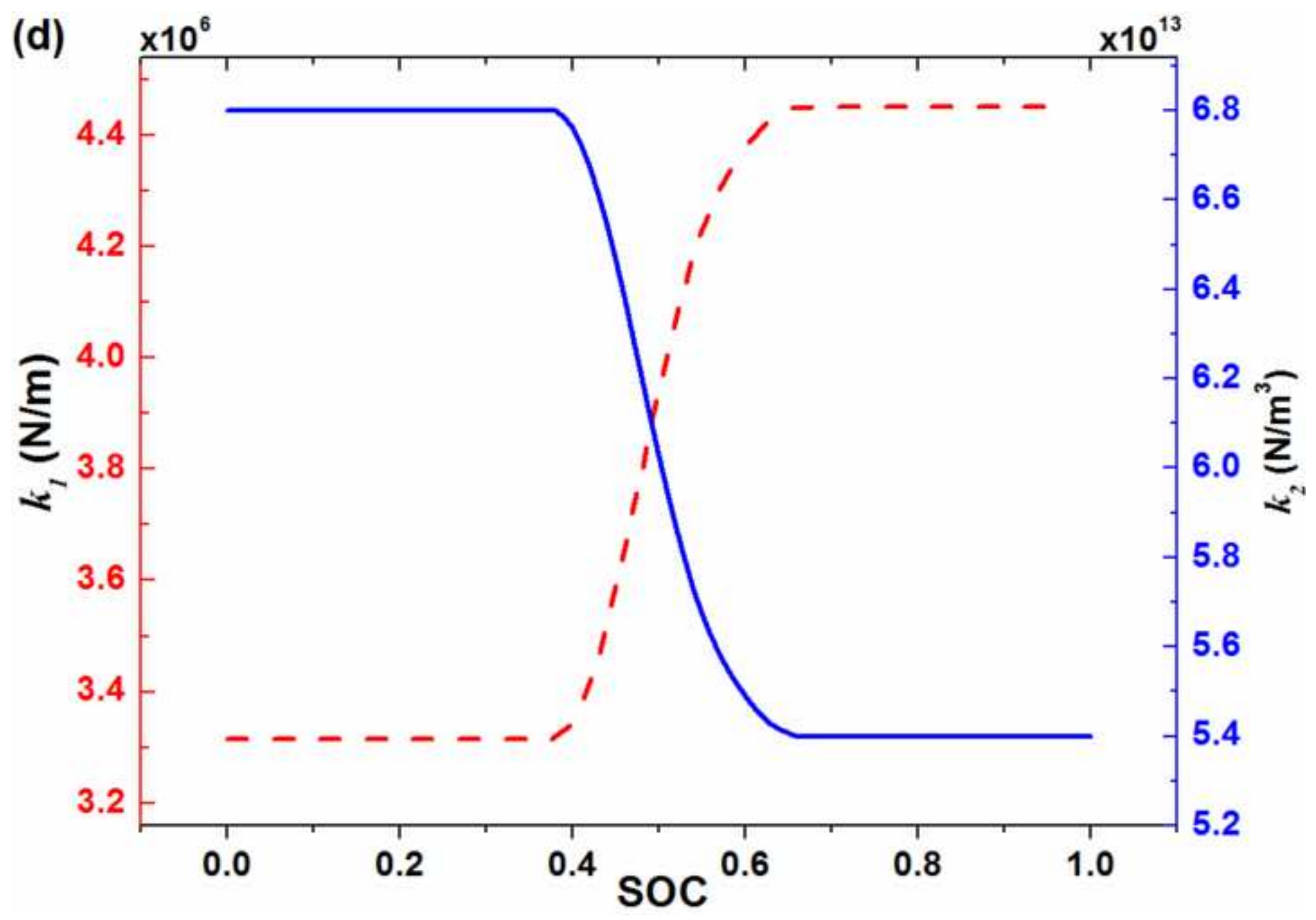




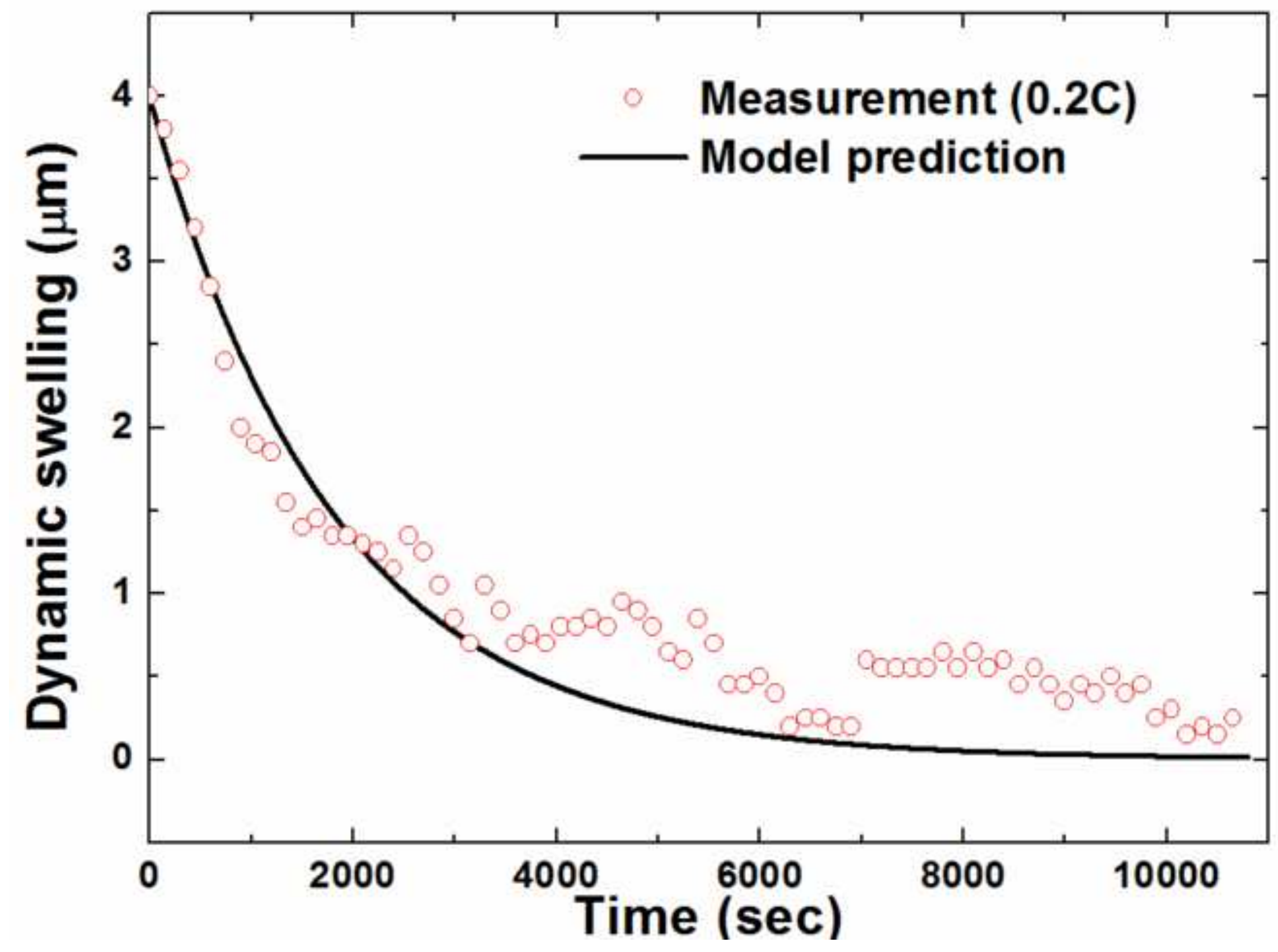



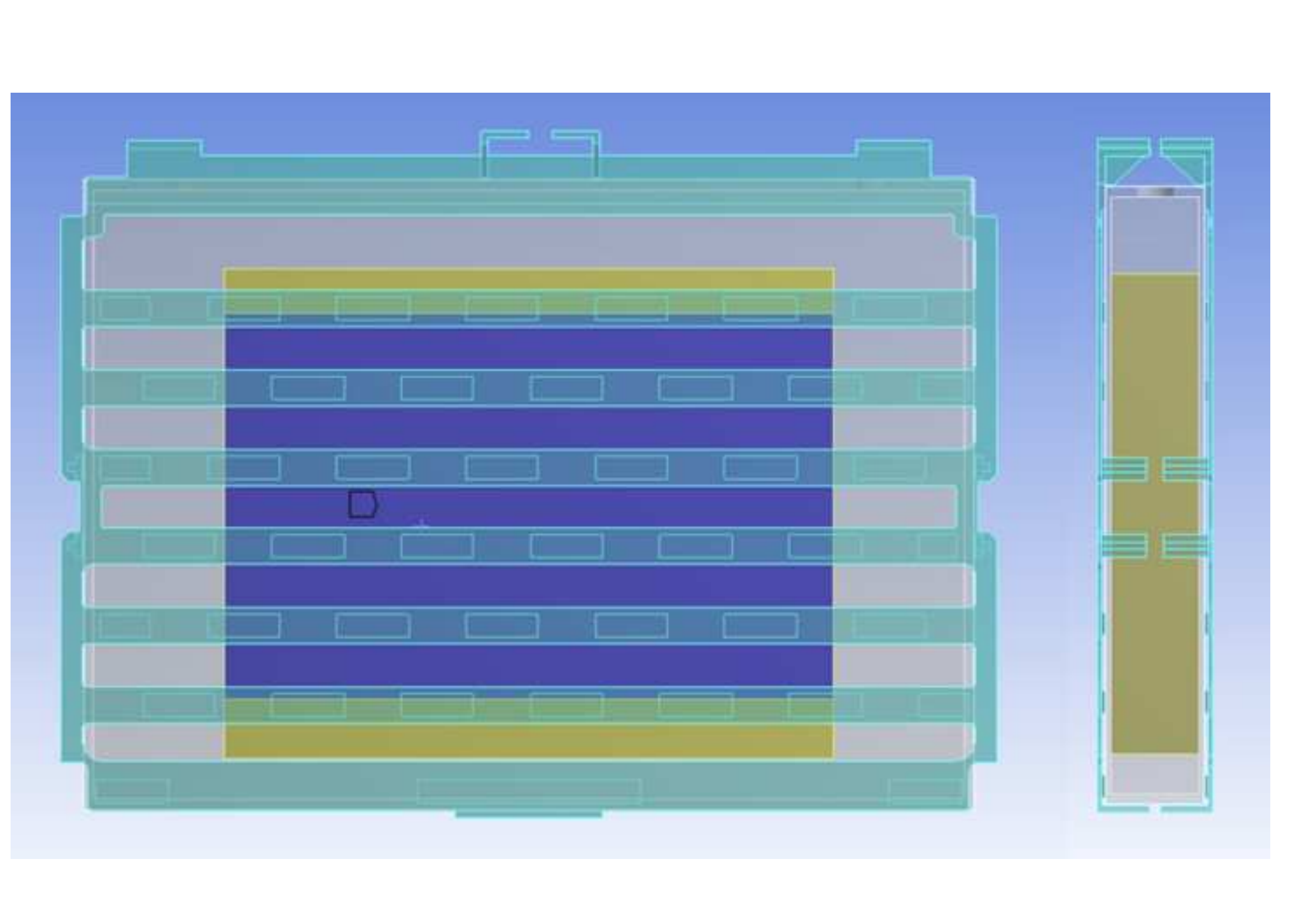

.

.
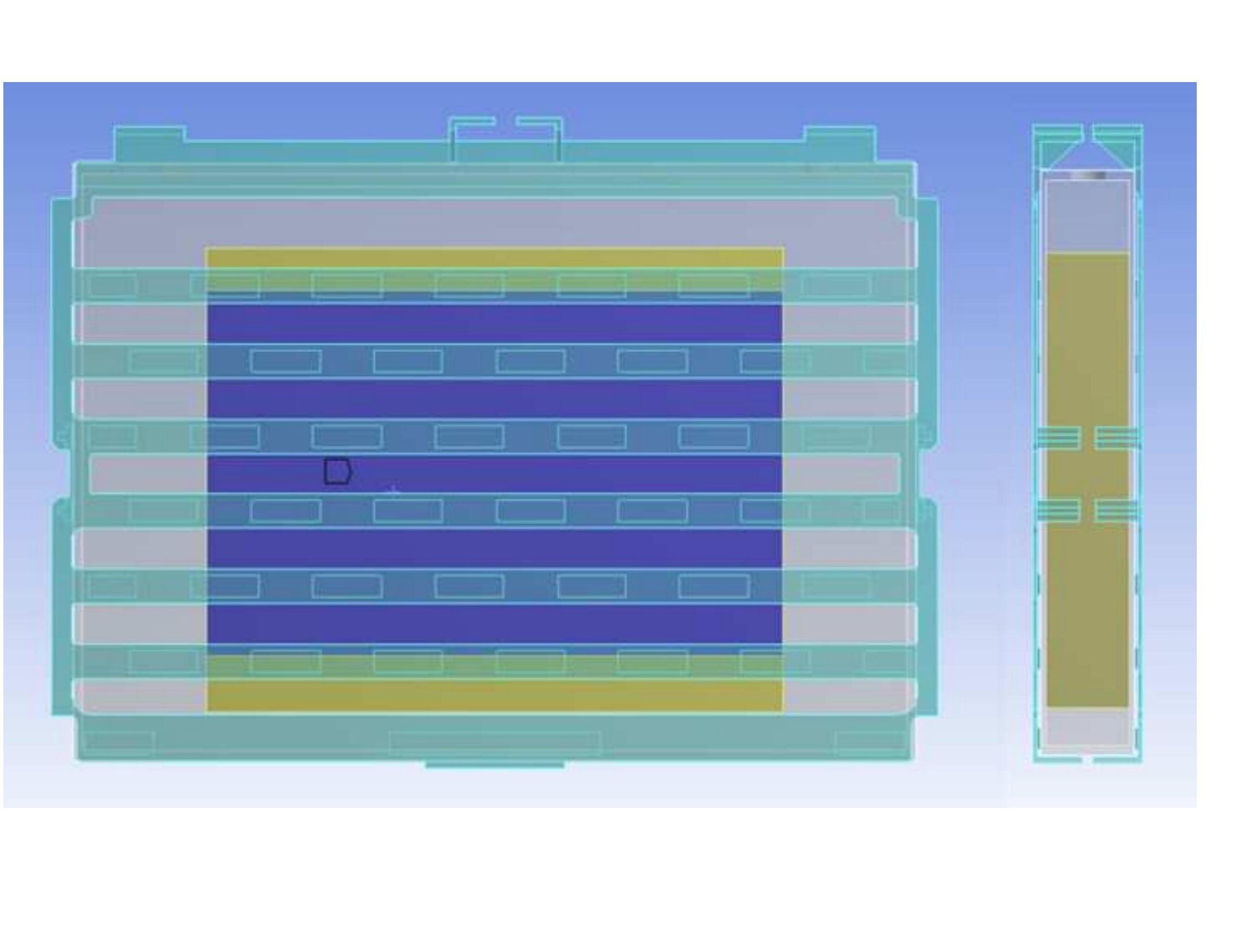


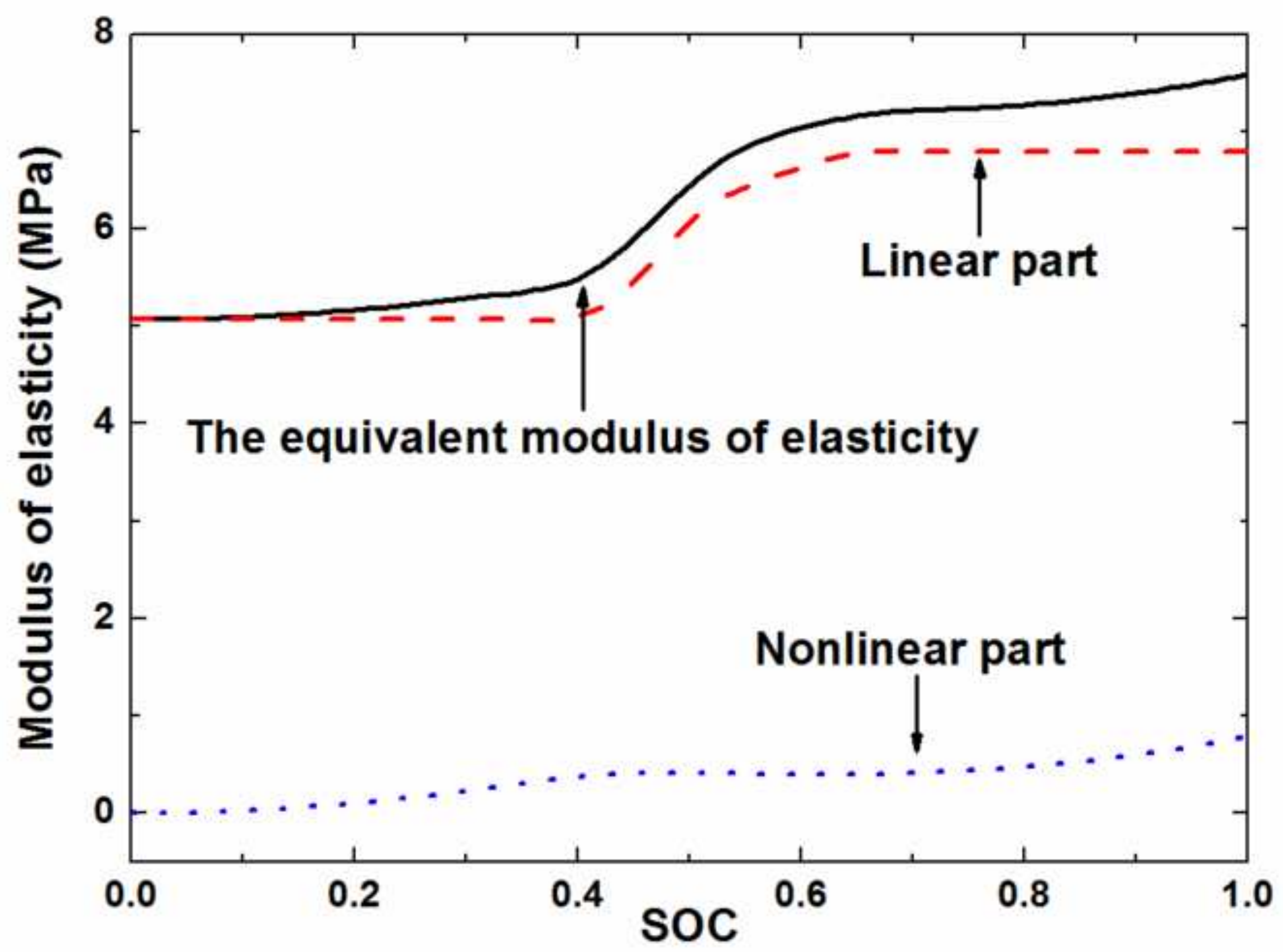




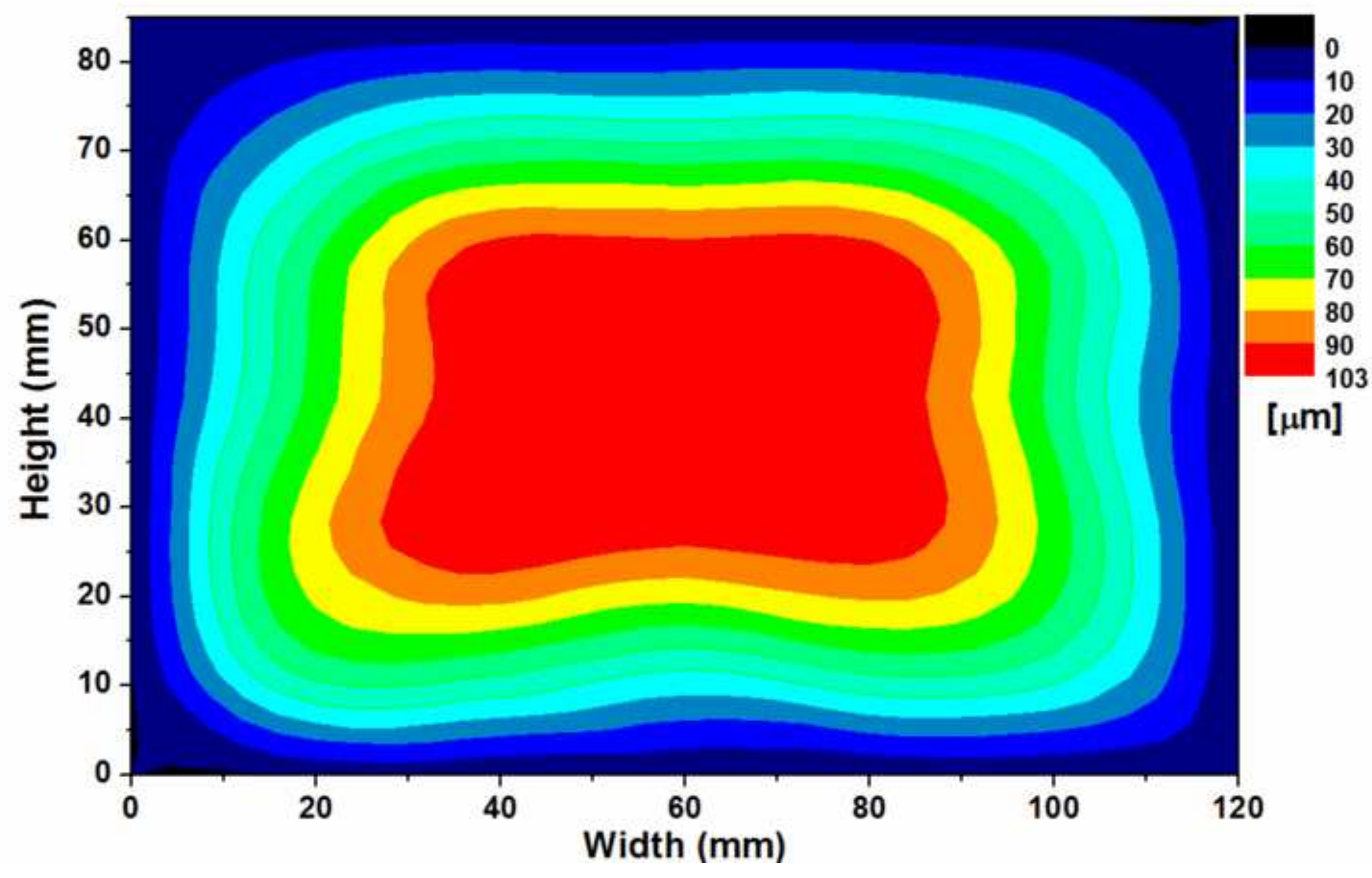




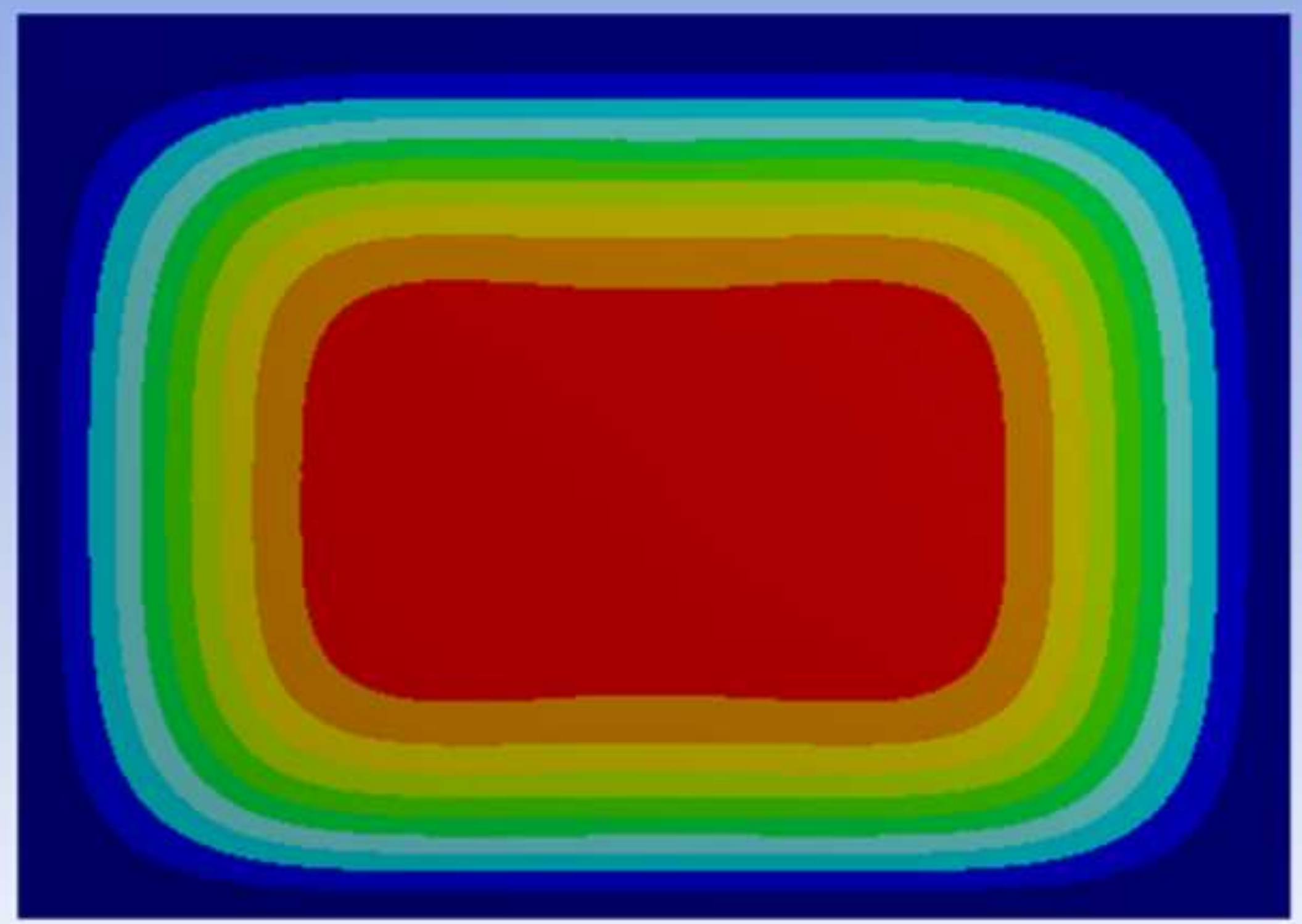

\begin{tabular}{|l|l}
\hline & 103 \\
90 \\
80 \\
70 \\
60 \\
50 \\
40 \\
30 \\
20 \\
10 \\
-1.3176 \\
{$[\boldsymbol{\mu m}]$}
\end{tabular}




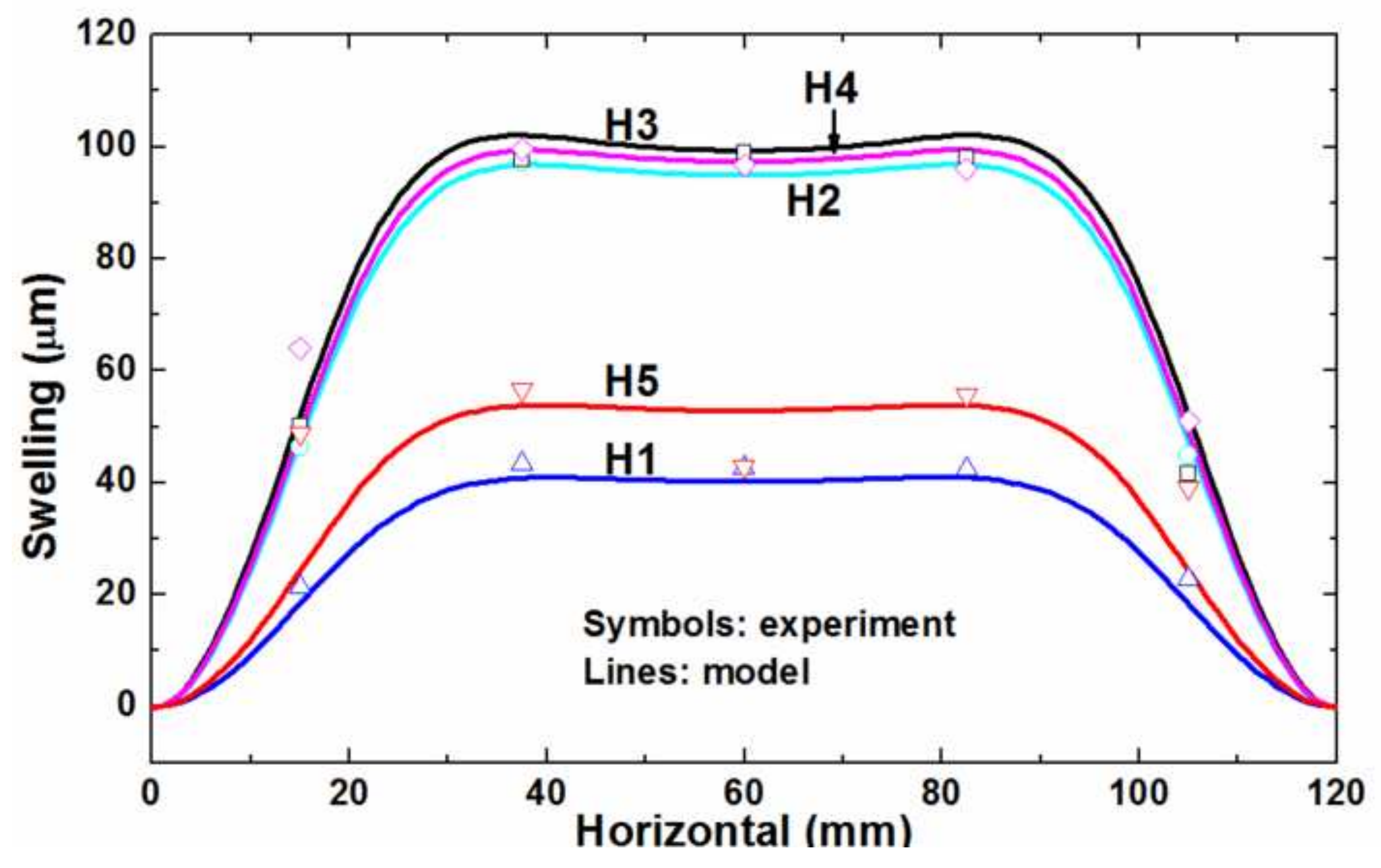




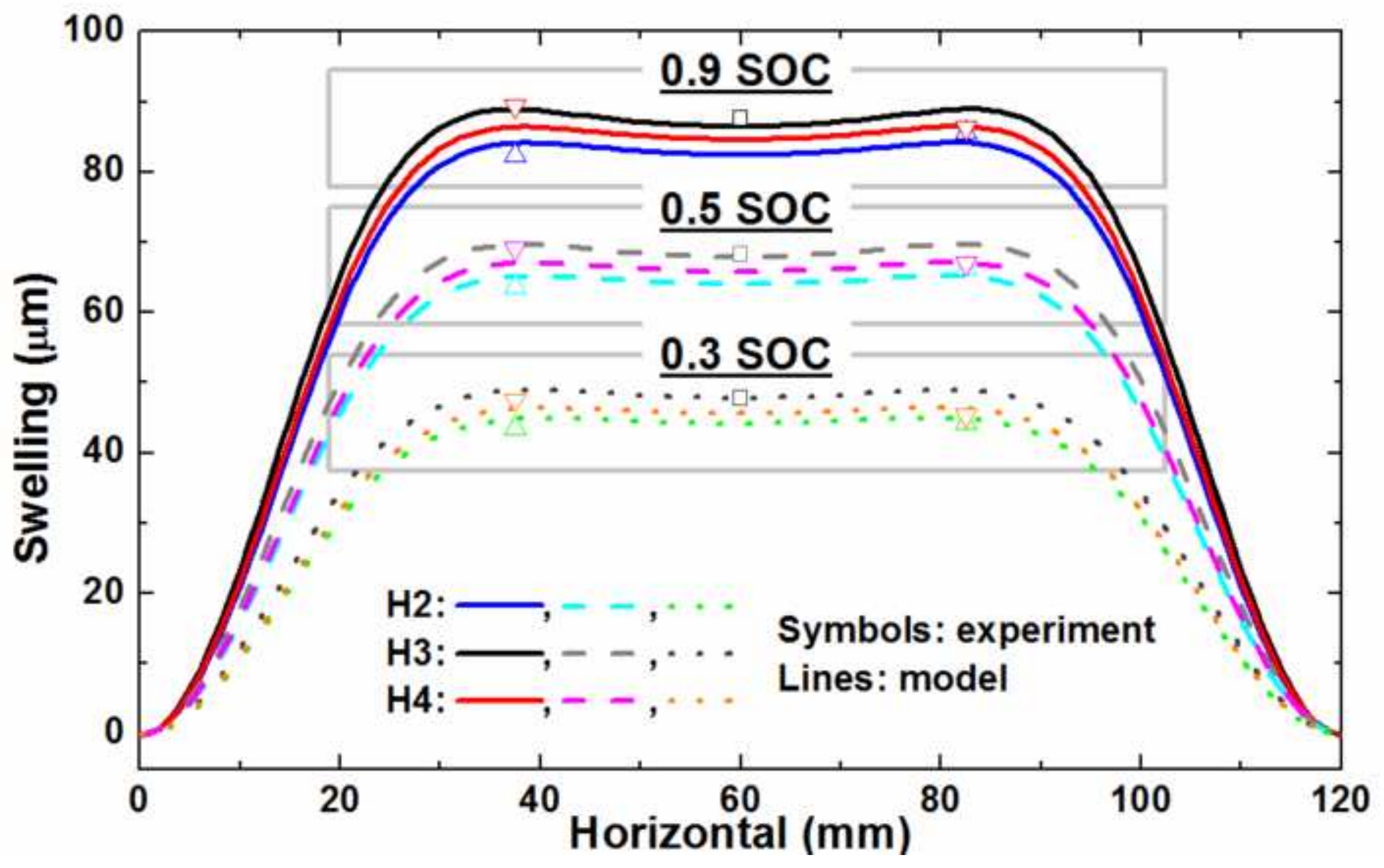




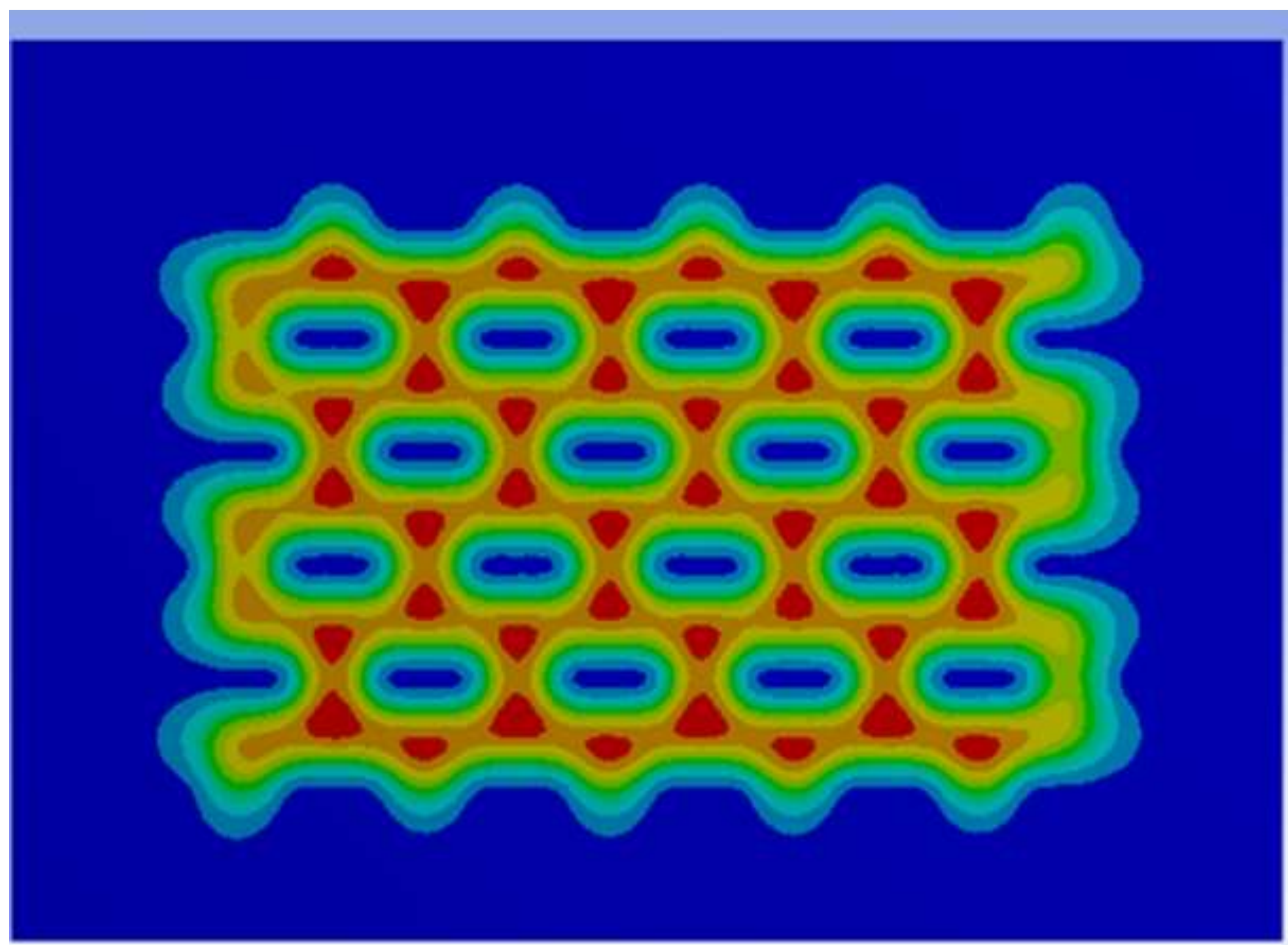

2.9 Max 2.6 2.2 1.9 1.5 1.2 0.81 0.45 0.096 $-0.26 \mathrm{Min}$ [ $\mu \mathrm{m}]$ 


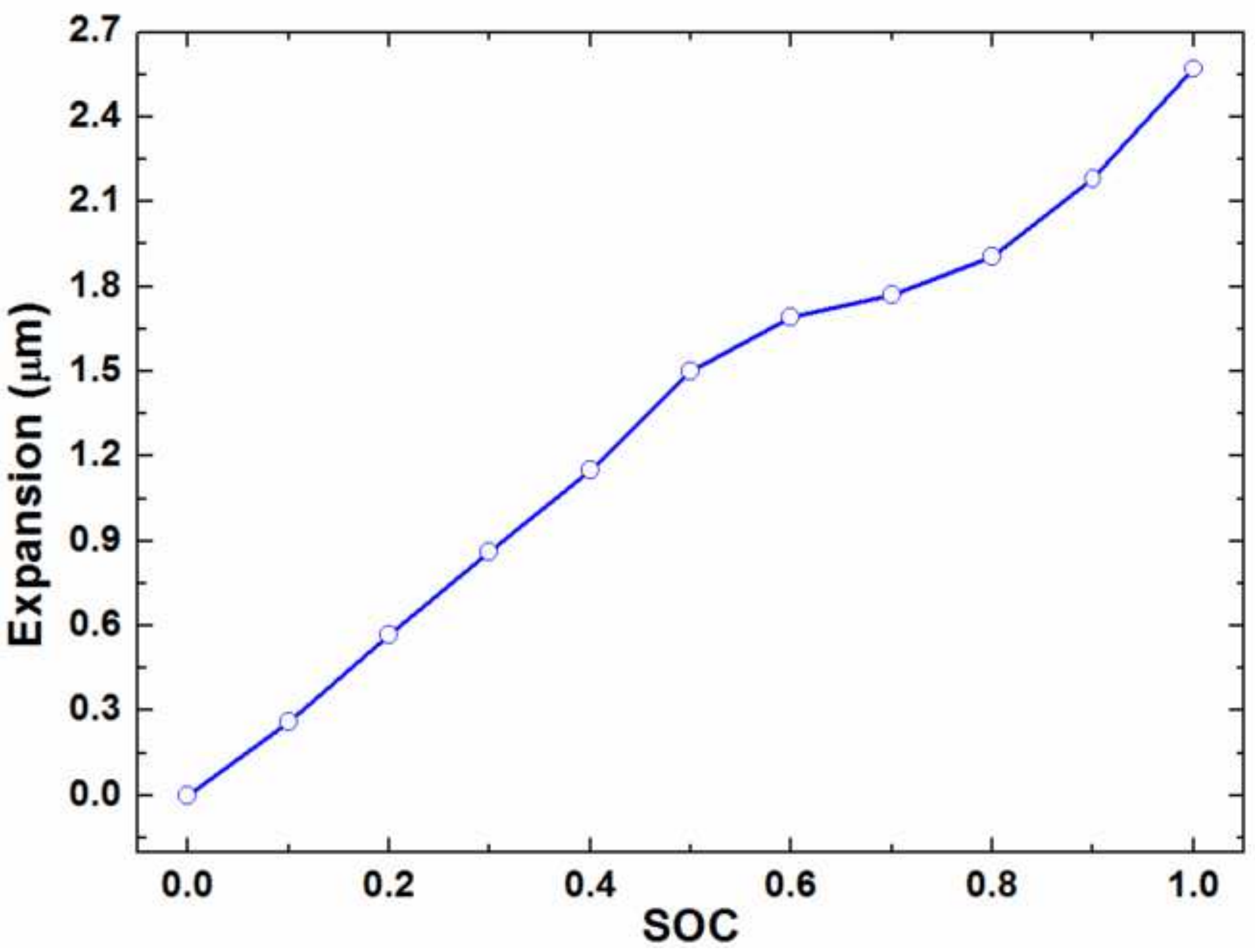




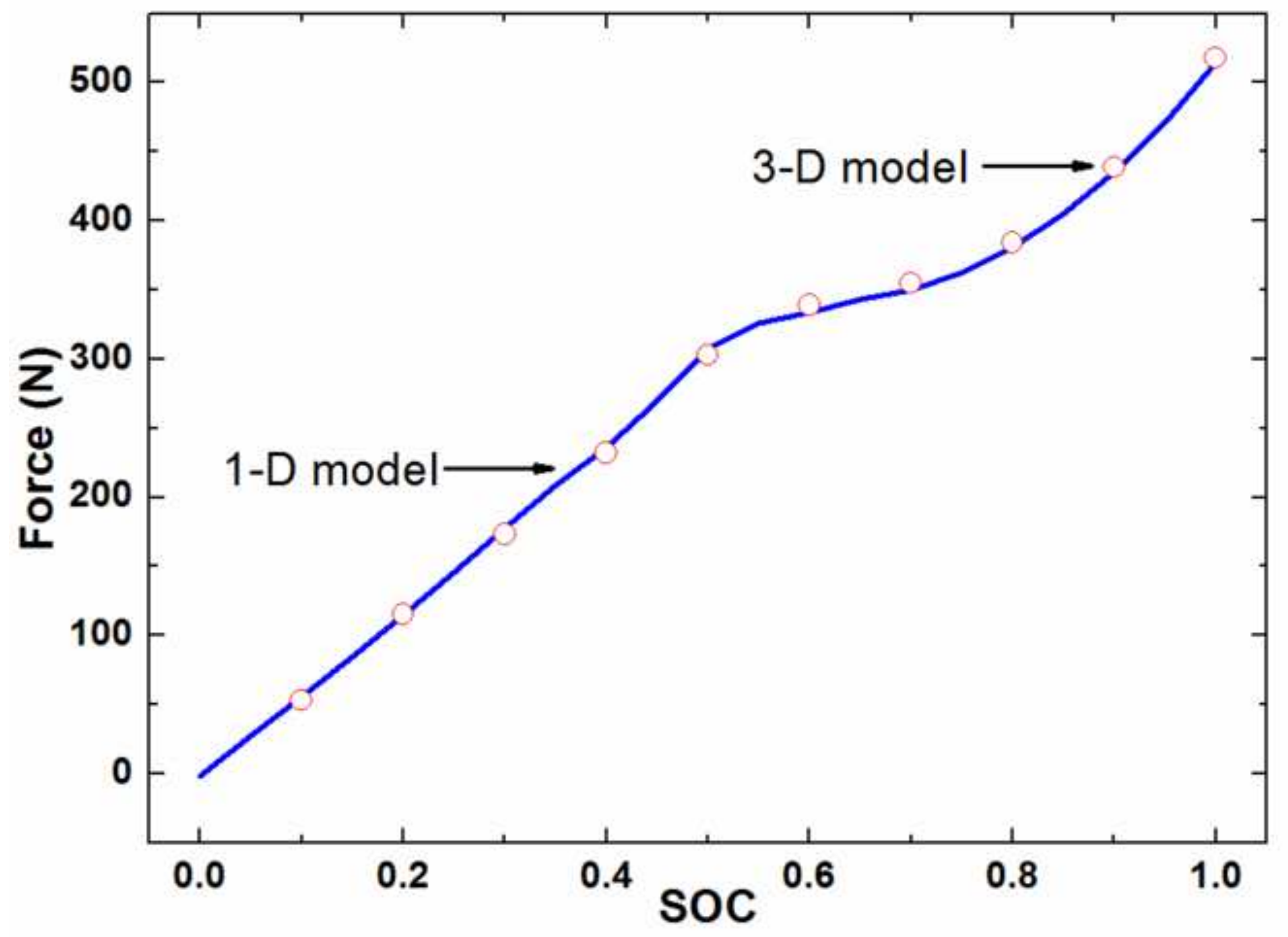




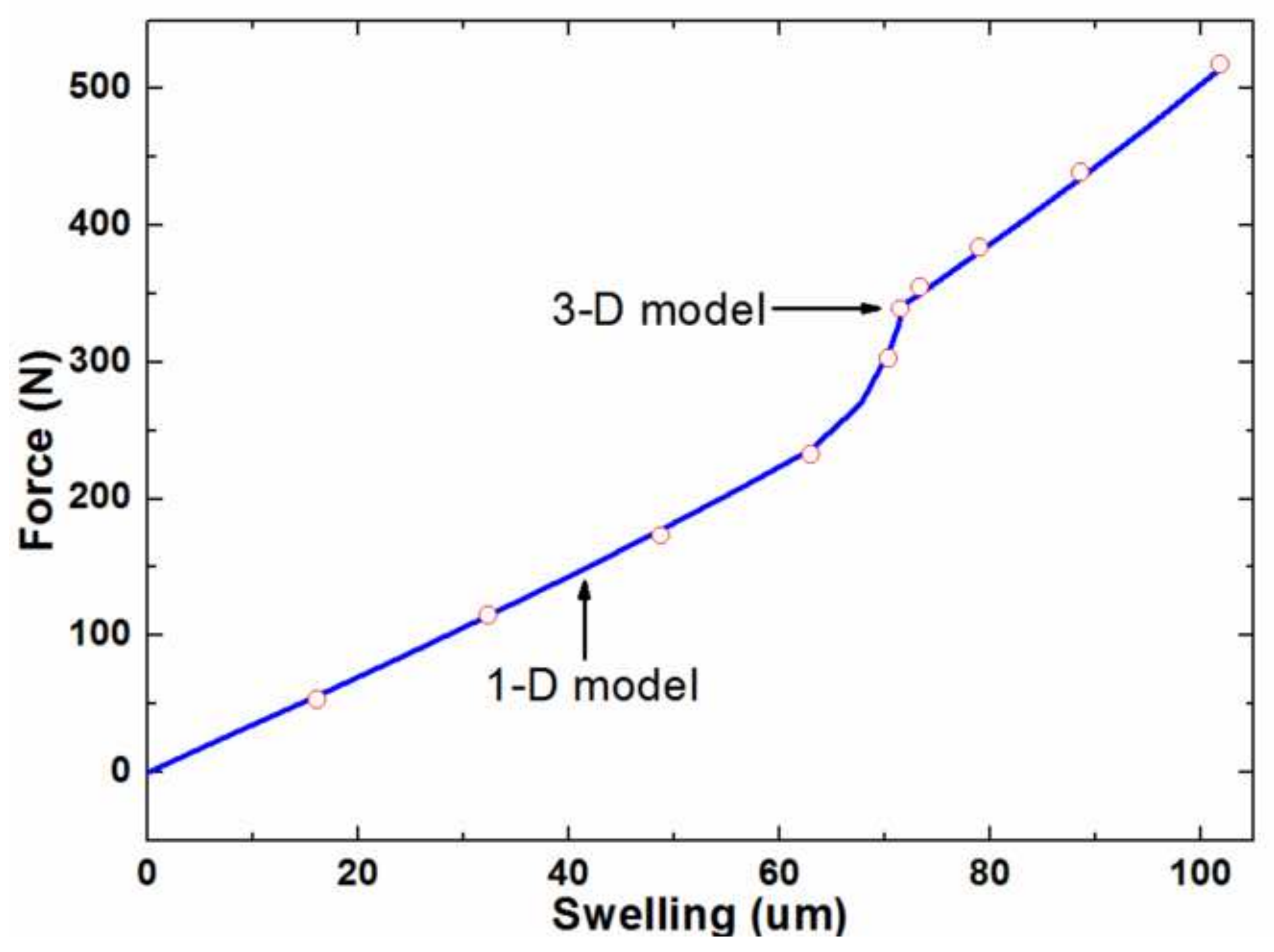




\section{Figure captions}

Figure 1. Schematic diagram of the experimental setup showing the fixture, the cell, and the 25 sensor locations labeled 1...25.

Figure 2. Forces for three cases: (a) free swelling of the battery cell due to Li-ion intercalation; (b) swelling of the battery cell and spacer under constraints; (c) constrained swelling of the battery cell and spacer due to Li-ion intercalation.

Figure 3. (a) The quasi-static compression force, quasi-static swelling, and dynamic (transient) swelling over SOC during discharge. (b) The quasi-static force versus swelling during discharge at several preloads. (c) The variation of $\alpha$ and $\beta$ over SOC. (d) The variation of equivalent stiffness values over SOC.

Figure 4. Measured dynamic swelling and simulation results with the phenomenological model during a relaxation period.

Figure 5. 3-D model of the battery cell. The yellow, semi-transparent white, and semi-transparent blue domains represent the jellyroll, the Aluminum case, and half of the spacer respectively. The purple domain represents the contact region between the jellyroll and the case.

Figure 6. The evolution of the equivalent modulus of elasticity for the jellyroll over SOC.

Figure 7. (Color online) Swelling shape on the surface of the battery cell due to Li-ion intercalation at fully charge state (1.0 SOC); (a) measurements; (b) model predictions, and (c) comparison between the 3-D phenomenological model (solid lines) and measurement (symbols) along the horizontal lines H1to H5 (locations of the horizontal lines are provided in Figure 1). (d) The comparison of free swelling at a variety of SOC between the 3-D model (solid, dashed, and dotted lines) and measured data (symbols).

Figure 8. (a) Constrained swelling on the surface at the fully charged state (1.0 SOC) and (b) Constrained swelling over SOC in the center region, at a location where there is no contact with the spacer. Comparison of force between the 1-D and the 3-D phenomenological models for a single battery cell without preload; (c) force versus SOC and (d) force versus swelling; the solid line shows the force estimated from the 1-D model, whereas the circles show the force estimated from the 3-D model. 